\title{
Experimental Validation of a Framework for the Design of Controllers that Induce Stable Walking in Planar Bipeds
}

\author{
E.R. Westervelt, G. Buche, and J.W. Grizzle
}

\begin{abstract}
This paper presents the experimental validation of a framework for the systematic design, analysis, and performance enhancement of controllers that induce stable walking in $\mathrm{N}$ link underactuated planar biped robots. Controllers designed via this framework act by enforcing virtual constraintsholonomic constraints imposed via feedback-on the robot's configuration which create an attracting two-dimensional invariant set in the full walking model's state space. Stability properties of resulting walking motions are easily analyzed in terms of a two-dimensional sub-dynamic of the full walking model. A practical introduction to and interpretation of the framework is given. In addition, the paper develops the ability to regulate the average walking rate of the biped to a continuum of values by modification of within-stride and stride-boundary characteristics, such as step length.
\end{abstract}

\section{Introduction}

The main factor contributing to the slow development of usable legged machines is the difficulty of simultaneously endowing legged machines with energy efficiency and stability, two essential attributes of any autonomous vehicle. The two means for addressing this problem are machine design and gait control. Through mechanical design, legged machines can be made efficient by using, for example, lighter materials and more efficient actuators. To enhance stability, legged machines can be designed to have morphologies that decrease the possibility of overturn: feet can be made larger and the number of legs increased. Through the use of control, a legged machine's gait may be designed and tuned to exploit the mechanism's natural dynamics to yield efficient locomotion. Control may also be used to impose gaits that, under some assumptions, have guarantees of stability. Typically, this has been accomplished by controlling the machine's motion to be slow to minimize inertial effects so that quasi-static stability measures may be used.

The past forty years have seen the development and construction of many mechanically sophisticated biped robot prototypes [2] that, to varying degrees of success, have addressed the efficiency-stability problem primarily through mechanism design. The development of equally sophisticated control

E.R. Westervelt is with the Department of Mechanical Engineering, The Ohio State University, Columbus, Ohio 43210-1154, USA (e-mail: westervelt.4@osu.edu)

G. Buche is with the Laboratoire d'Automatique de Grenoble, Ecole Nationale d'Ingénieurs Electriciens de Grenoble, BP 46, 38402 St Martin d'Hères, FRANCE (e-mail: Gabriel.Buche@ inpg.fr)

J.W. Grizzle is with the Control Systems Laboratory, Electrical Engineering and Computer Science Department, University of Michigan, Ann Arbor, Michigan 48109-2122, USA (e-mail: grizzle@umich.edu) algorithms that address the efficiency-stability problem which induce walking in these prototypes has not kept pace. This is evidenced by the reliance on heuristics, such as the zero moment point ${ }^{1}$ (ZMP) principle [9], [30].

It is conjectured here that the development of control algorithms that induce truly dynamic biped walking has been slow for five reasons that are inherent to dynamic biped walking. The five difficulties are divided into two groups. The first three difficulties are common to all biped walking models while the last two are common only to dynamic biped walking.

The first difficulty is limb coordination. Bipeds are typically high DOF mechanisms, but the task of biped walking is inherently a low DOF task. That is, bipeds typically have many links and joints that must be coordinated to achieve locomotion - the moving of the robot's center of mass (COM) from one point to another. The second difficulty is effective underactuation during the single support phase (the phase of walking when only one leg is touching the ground). Unlike traditional robotic manipulators which are securely fastened to the environment, bipeds are designed to move with respect to the environment. Unilateral constraints severely limit the amount of torque that may be supplied at the stance leg ankle joint; because of finite foot size, too large a torque supplied at the angle joint results in foot roll-over. This has been recognized in [7], [9], [14], [18]. The third difficulty is hybrid dynamics. The presence of impacts and changing dynamic constraints during the walking cycle due to foot touchdown and lift-off necessarily leads to models that are hybrid.

The last two difficulties are common only to dynamic biped walking. The first is static instability of the biped during portions of the walking cycle. That is, in dynamic walking during portions of the walking cycle the projection of the biped's $\mathrm{COM}$ - and usually the ZMP—onto the walking surface is outside of the biped's polygon of support. This prohibits the use of the popular ZMP principle to ensure stability. The second, and final, difficulty is the design of limit cycles. Dynamically stable walking corresponds to the existence of limit cycles in the biped's state space. The design of controllers that induce limit cycles, while a challenge in its own right, is made significantly more difficult by the first four difficulties and by the need for energy efficiency.

The approach of this work (developed in [12], [33], [34]) has been to study a class of biped robots whose model is only as complex as required to capture these inherent difficulties. The class consists of planar biped robots that are $N$-link-and thus have $N$ DOF during the swing phasewith walking taking place in the sagittal plane (the plane

\footnotetext{
${ }^{1}$ In the legged robotics literature, the center of pressure (COP) - the point on the ground where the resultant of the ground-reaction force acts-is often referred to as the zero moment point [9].
} 


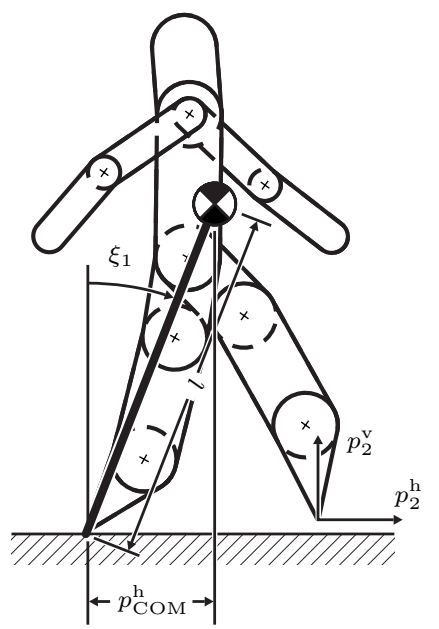

Fig. 1. A higher DOF planar robot model with its center of mass labeled. Cartesian coordinates are indicated at the swing leg end. The COM is of mass $M$ with inertia $J$.

that divides the body into left and right halves; see Figure 1. Restricting attention to the plane where forward progression is achieved (the sagittal plane) is reasonable since the sagittal plane dynamics are almost decoupled from those in the frontal plane (the plane that divides the body into front and back halves) in that stability in the frontal plane can be achieved with only frontal plane control actions, such as step width control [1], [20]. The model assumes point feet with no actuation between the stance leg end and the ground and full actuation at all internal joints. The model for the swing phase of walking is therefore that of an underactuated mechanical system. Without feet, the ZMP heuristic is not applicable, and thus the effective underactuation must be explicitly addressed in the feedback control design; see Figure 2. The phase of double support (when both legs are on the ground) is assumed to be instantaneous and is modeled by a rigid contact model [15].

One of the fruits of studying a biped model of minimal complexity has been the development of a rigorous framework for the systematic analysis and design of efficient, dynamic, stable walking motions [12], [34]. It is anticipated that the results for a robot with point feet will lead to an equally complete control theory for walking with anthropomorphic foot action.

In addition to walking at a fixed rate, this framework provides two additional control features: 1) the ability to compose such controllers to obtain walking at several discrete walking rates with guaranteed stability during the transitions; and 2) the ability to regulate the average walking rate to a continuum of values by modification of within-stride characteristics [33].

The purpose of this paper is to provide experimental validation of this framework on the biped robot prototype, RABBIT constructed by the French project Commande de Robots à Pattes of the CNRS - GdR Automatique [3], [4]. In presenting the experimental validation, several implementation issues will be discussed, and the control design framework will be interpreted. The final contribution of this paper is to develop a technique to regulate the average walking rate to a continuum of values by modification of within-stride and stride-boundary

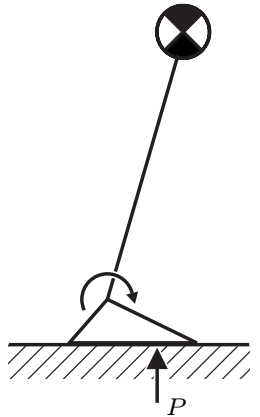

(a)

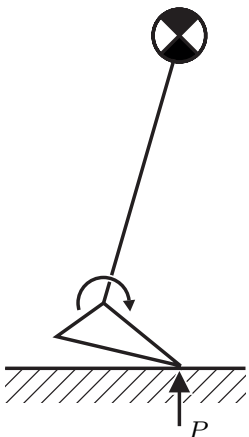

(b)
Fig. 2. The zero moment point (ZMP) principle according to Goswami [9]. Idealize a robot in single support as a planar inverted pendulum attached to a base consisting of a foot with torque applied at the ankle. Assume adequate friction so that the foot is not sliding. In (a), the robot's nominal trajectory has been planned so that the center of pressure of the forces on the foot, $P$, remains strictly within the interior of the footprint. In this case, the foot will not rotate (i.e, the foot is acting as a base, as in a normal robotic manipulator), and thus the system is fully actuated. It follows that small deviations from the planned trajectory can be attenuated via feedback control, proving stabilizability of the walking motion. In case (b), however, the center of pressure has moved to the toe, allowing the foot to rotate. The system is now underactuated (two degrees of freedom and one actuator), and designing a stabilizing controller is nontrivial, especially when impact events are taken into account. The ZMP principle says to design trajectories so that case (a) holds; i.e., walk flat footed. This leads to awkward, quasi-static walking motions.

characteristics, such as step length. This result is a nontrivial extension to the event-based PI-control result given in [33] and was developed in response to the within-stride scheme's inability to effectively regulate average walking rate on the prototype.

The paper is structured as follows: Section 2 describes the prototype RABBIT, gives the model for walking of [34] specialized to RABBIT, and describes three issues associated with RABBIT's construction which were addressed in the control implementation. Section 3 summarizes, interprets, and gives the experimental implementation of the control framework presented in [34]. Section 4 reports on the experimental validation of the control implementation for walking at a fixed rate described in Section 3.4 and the experimental validation of the transitioning technique presented [33] and the event-based control for the regulation of average walking rate developed in Appendix 2.

\section{The prototype RABBIT}

The five-link, planar prototype RABBIT (see Figure 3) is located at the Laboratoire D'Automatique de Grenoble in Grenoble, France. It was constructed jointly by several French research laboratories, spanning Mechanical Engineering, Automatic Control, and Robotics [3], [4]. The RABBIT project was initiated in 1997 and is funded by the French CNRS and the French National Research Council. Its central mission is to build a prototype for studying truly dynamic motion control. In particular, the mechanism was designed to allow for high speed walking and running. In its mechanical design complexity was 


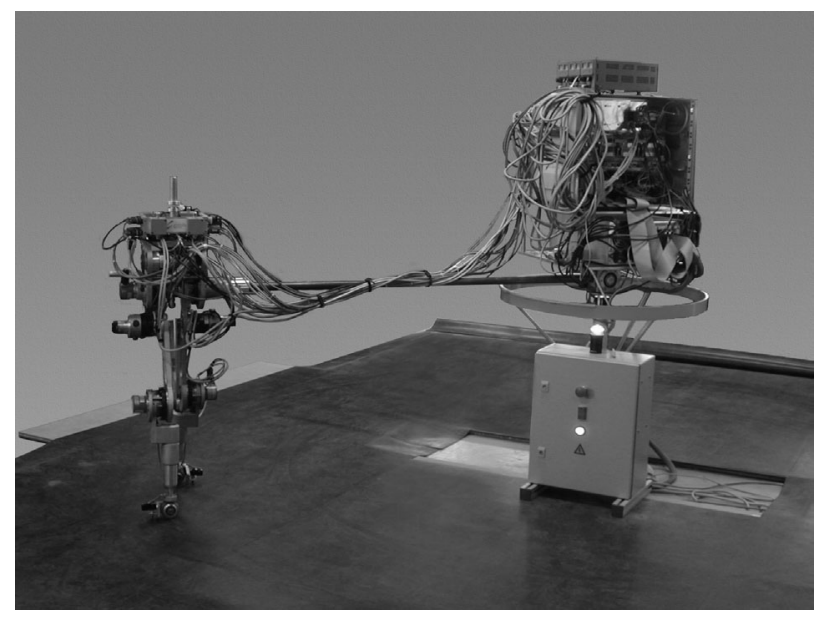

Fig. 3. The biped prototype RABBIT's experimental setup.

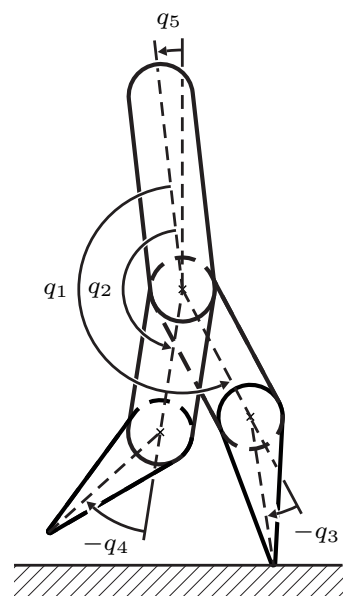

(a)

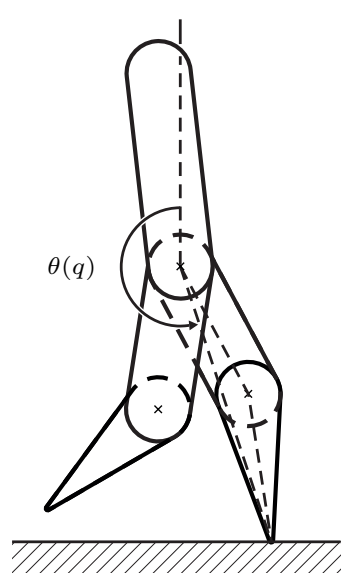

(b)
Fig. 4. Schematic of the prototype RABBIT with measurement conventions.

included only as required to accurately model biped walking. (The theoretical development given in [12], [34] was motivated by a desire to control RABBIT.)

RABBIT's five links are connected by revolute joints that form two symmetric legs and a torso; see Figure 4(a). Actuators supply torque between each of the four internal joints: one at each knee and one between the torso and each femur. All actuators are identical and capable of producing a peak torque of $150 \mathrm{Nm}$. To prevent motions in the frontal plane, RABBIT was constructed with a boom attached at the hip; see Figure 3. RABBIT has no feet and no means of supplying actuation between the stance leg end and the ground.

The link parameter values were identified by a group associated with the project and are given in Table 1 with the measurement conventions given in Figure 5. To obtain configuration information, encoders are located at each internal joint giving the robot's shape, and between the boom and hip giving the robot's orientation with respect to a world frame. Binary contact switches located at the leg ends are used to detect whether or not a leg is in contact with the walking
Table 1. Identified link parameters for RABBIT.

\begin{tabular}{|c|c|c|c|}
\hline Model parameter & Units & Label & Value \\
\hline \multirow{3}{*}{ Mass } & \multirow{3}{*}{$\mathrm{kg}$} & $M_{T}$ & 12 \\
\hline & & $M_{f}$ & 6.8 \\
\hline & & $M_{t}$ & 3.2 \\
\hline \multirow{3}{*}{ Length } & \multirow{3}{*}{$\mathrm{m}$} & $l_{T}$ & 0.63 \\
\hline & & $l_{f}$ & 0.4 \\
\hline & & $l_{t}$ & 0.4 \\
\hline \multirow{3}{*}{ Inertia } & \multirow{3}{*}{$\mathrm{m}^{2} \mathrm{~kg}$} & $I_{T}$ & 1.33 \\
\hline & & $I_{f}$ & 0.47 \\
\hline & & $I_{t}$ & 0.20 \\
\hline \multirow{3}{*}{ Mass center } & \multirow{3}{*}{$\mathrm{m}$} & $p_{T}^{M}$ & 0.24 \\
\hline & & $p_{f}^{M}$ & 1.11 \\
\hline & & $p_{t}^{M}$ & 0.24 \\
\hline \multirow[b]{2}{*}{ Viscous friction } & \multirow[b]{2}{*}{ Ns } & $F_{\mathrm{v}, \mathrm{H}}$ & 16.5 \\
\hline & & $F_{\mathrm{v}, \mathrm{K}}$ & 5.48 \\
\hline \multirow{2}{*}{ Static friction } & \multirow{2}{*}{$\mathrm{Nm}$} & $F_{\mathrm{s}, \mathrm{H}}$ & 15.0 \\
\hline & & $F_{\mathrm{S}, \mathrm{K}}$ & 8.84 \\
\hline Gear ratio & - & $n_{\mathrm{g}}$ & 50 \\
\hline Motor rotor inertia & $\mathrm{m}^{2} \mathrm{~kg}$ & $I_{\mathrm{a}}$ & 0.83 \\
\hline
\end{tabular}

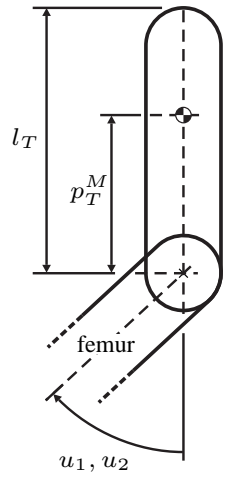

(a) Schematic of torso

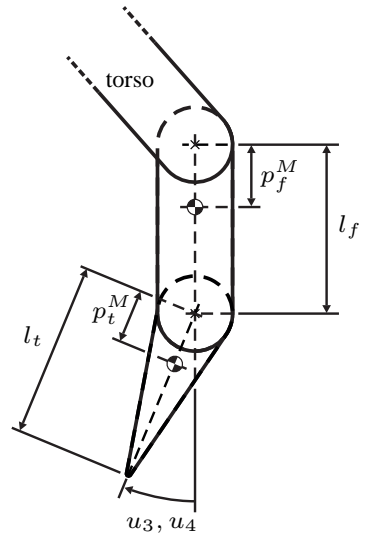

(b) Schematic of leg.
Fig. 5. Schematic of RABBIT's link parameter measurement conventions.

surface.

For a real-time control platform, RABBIT uses a dSPACE DS1103 system. With the DS1103 system, run-time software is created by automatic translation and cross-compiling of Simulink diagrams for the system's $400 \mathrm{MHz}$ PowerPC 604e DSP, allowing the real-time controller software to be developed in a high-level language. This obviates the need for lowlevel I/O programming and facilitates debugging. In addition, the system provides low-level computation, digital-to-analog and analog-to-digital conversion, as well as a user interfaceall in a single package.

\subsection{The mathematical model of RABBIT walking}

The model of RABBIT walking used for controller design assumes that all motions take place in the sagittal plane and that a normal gait consists of symmetric, alternating phases 
of single support and double support (see Appendix 1 for a complete list of hypotheses). These two phases of the walking cycle naturally lead to a mathematical model of the biped consisting of two parts: the differential equations describing the dynamics during the single support phase, and a discrete model of the dynamics of the double support phase. The biped model is thus hybrid in nature, consisting of continuous dynamics and a re-initialization rule at the contact event.

\subsubsection{Swing phase model: a nonlinear underactuated con- trol system}

The dynamic model of RABBIT during the swing phase is that of an underactuated, planar five-link inverted pendulum. Let $q:=\left(q_{1}, \cdots, q_{5}\right)^{\prime}$ be the set of angular coordinates describing the configuration of the robot, given in Figure 4(a). Since only symmetric gaits are of interest here, the same model can be used irrespective of which leg is the stance leg if the coordinates are re-labeled after each phase of double support.

The torques $u_{i}, i=1$ to 4 are applied between torso and femurs and between the femurs and the tibias. Using the method of Lagrange and defining the state as $x:=\left(q^{\prime}, \dot{q}^{\prime}\right)^{\prime}$, the model is

$$
\begin{aligned}
\dot{x} & =\left[\begin{array}{c}
\dot{q} \\
D^{-1}(q)[-C(q, \dot{q}) \dot{q}-G(q)+B u]
\end{array}\right] \\
& =: \quad f(x)+g(x) u
\end{aligned}
$$

The state space of the model is taken as $T \mathcal{Q}:=\{x=$ $\left.\left(q^{\prime}, \dot{q}^{\prime}\right)^{\prime} \mid q \in \mathcal{Q}, \dot{q} \in \mathbb{R}^{5}\right\}$, where $\mathcal{Q}$ is a simply-connected, open subset of $[0,2 \pi)^{2} \times[-\pi, \pi)^{3}$ corresponding to physically reasonable configurations of the robot (for example, with the exception of the end of the stance leg, all points of the robot being above the walking surface; one could also impose that the knees are not bent backward, etc.).

\subsubsection{Double support model: a rigid impact}

The double support phase-the transition from one leg to another-is modeled with a rigid impact map. The rigid contact model collapses the double support phase to an instant in time resulting in a discontinuity in the velocity component of the state, with the configuration remaining continuous. In addition to modeling the change in state of the robot, the impact model accounts for the relabeling of the robot's coordinates that occurs after each phase of double support.

The result of the impact and relabeling of the states is then an expression

$$
x^{+}=\Delta\left(x^{-}\right)
$$

where $x^{+}:=\left(q^{+}, \dot{q}^{+}\right)$(resp. $\left.x^{-}:=\left(q^{-}, \dot{q}^{-}\right)\right)$is state value just after (resp. just before) impact and

$$
\Delta\left(x^{-}\right):=\left[\begin{array}{c}
\Delta_{q} q^{-} \\
\Delta_{\dot{q}}\left(q^{-}\right) \dot{q}^{-}
\end{array}\right]
$$

where $\Delta_{q}$ and $\Delta_{\dot{q}}\left(q^{-}\right)$are linear maps (see Appendix 1 for a complete list of hypotheses assumed for this rigid impact model).

\subsubsection{The complete model for walking: a hybrid nonlinear underactuated control system}

With the addition of an appropriately chosen switching set, $S$, the swing phase model can be combined with the double support model and expressed as a nonlinear system with impulse effects [12], [36]

$$
\begin{aligned}
\dot{x} & =f(x)+g(x) u & & x^{-} \notin S \\
x^{+} & =\Delta\left(x^{-}\right) & & x^{-} \in S .
\end{aligned}
$$

Here the switching set is chosen to be the set where both legs are on the ground,

$$
S:=\left\{(q, \dot{q}) \in T \mathcal{Q} \mid p_{2}^{\mathrm{v}}(q)=0, p_{2}^{\mathrm{h}}(q)>0\right\},
$$

and $x^{-}(t):=\lim _{\tau / t} x(\tau)$. The value of $p_{2}^{\mathrm{h}}(q)$ is taken to be positive so that for $x \in S$ the swing leg end is in front of the stance leg at the end of a step (a step is only defined when the swing leg progresses from behind to in front of the stance leg). Dynamically stable walking corresponds to the existence of a limit cycle in the state space of (5).

\subsection{Implementation issues}

This section presents three important aspects of RABBIT that are not addressed by the model given in the previous subsection. The effects are the additional dynamics introduced by the boom used to constrain RABBIT's motions to be planar, RABBIT's gear reducers, and RABBIT's irregular, non-rigid walking surface. These effects were accommodated in the design of controllers for the experiments presented in Section 4 so that the closed-loop system's experimental dynamic performance would more closely match the design specification.

\subsubsection{Constraining RABBIT to be planar}

The boom attached to RABBIT's hip constrains RABBIT's motions to the sagittal plane and constrains the sagittal plane to be tangent to a sphere centered at the universal joint that connects the boom to the center stand (see Figures 3 and 6). RABBIT therefore walks in a circle whose radius is determined by the length of the boom. The boom system consists of the boom, center stand, counterweight, and cabling. "Training wheels," shown in Figure 11, but not drawn in Figure 6, were attached to the boom to provide a measure of safety. The training wheels' post has a prismatic joint with a stop to prevent the robot's hip from dropping too low but otherwise does not support the robot's weight. The boom system also includes two encoders at the universal joint to measure horizontal and vertical angular displacement of the boom about the center stand. A boom system of this sort was also used for MIT Leg Lab's Spring Flamingo [24], as well as several of their other robots. The other typical means of constraining a biped robot's motion to be planar is through the use of wide feet. This idea was used in the design of Kenkyaku [6] and Meltran II [18], among others. The advantage of a boom system over wide feet is that a boom is able to constrain the robot's motion even when none of the feet are on the ground. This is important in the case of RABBIT, as one 


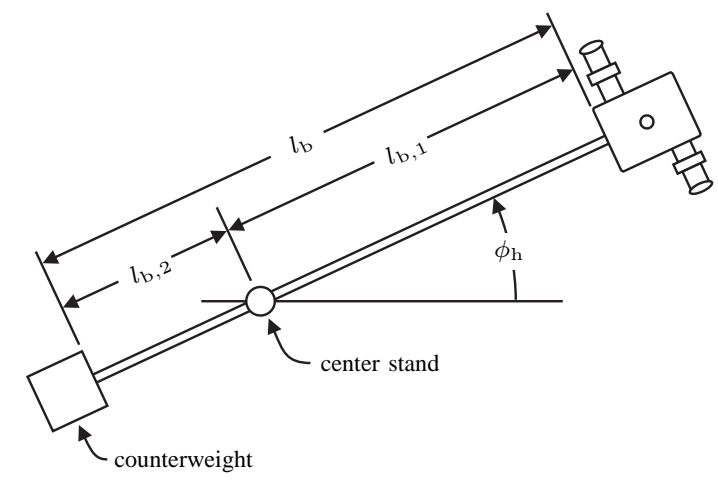

(a) Overhead view of RABBIT'S experimental setup. For clarity, the electronics are not drawn.

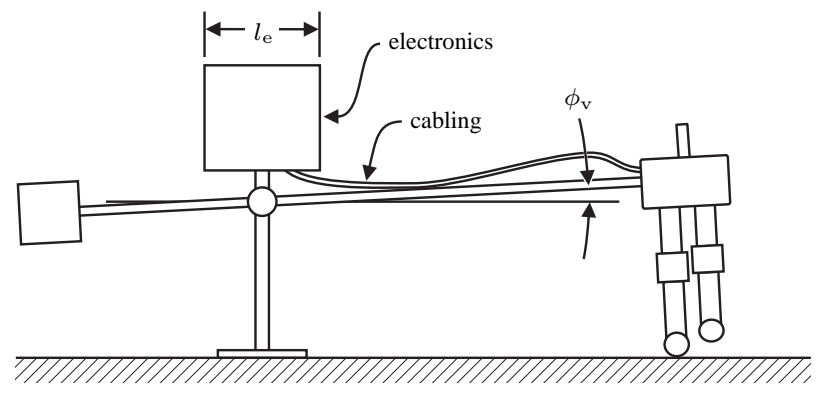

(b) Side view of RABBIT's experimental setup.

Fig. 6. Various dimensions of RABBIT's experimental setup.

of its stated purposes is to study running, which necessarily has a ballistic phase (when no feet are in contact with the ground). The use of a boom, however, requires some means of allowing the legs to move radially when in contact with the ground. To accomplish this in RABBIT, wheels aligned with the frontal plane were attached at the leg ends. Another consideration with a boom system is how to connect power and communications cabling to the experimental setup. Unless a slip ring is used, cabling connected to the experimental setup will become twisted or wound as the robot makes laps. Unfortunately, a slip ring was not installed at the time when the experiments reported here were performed, and RABBIT had to be "unwound" after each experiment.

The inertia of the boom system used to constrain RABBIT's motion to be planar results in additional inertia that is significant enough to require incorporation into RABBIT's model. The inertia has four components due to 1) the boom connecting RABBIT, the center stand, and the counterbalance, 2 ) the counterbalance, 3) the cabling connecting RABBIT to the support electronics, and 4) the support electronics (see Figures 3 and 6). Since the training wheels are not always used, and since they are relatively light, their inertia is not included. The inertia may be approximated as

$$
I_{\mathrm{s}}=\underbrace{\frac{1}{3} \frac{m_{\mathrm{b}}}{l_{\mathrm{b}}}\left(l_{\mathrm{b}, 1}^{3}+l_{\mathrm{b}, 2}^{3}\right)}_{\text {boom }}+\underbrace{m_{\mathrm{w}} l_{\mathrm{b}, 2}^{2}}_{\text {counterbalance }}+\underbrace{\frac{1}{3} m_{\mathrm{c}} l_{\mathrm{b}, 1}^{2}}_{\text {cabling }}
$$

Table 2. RABBIT's experimental platform parameters.

\begin{tabular}{c|c|c|c}
\hline Model parameter & Units & Label & Value \\
\hline \hline Constraint boom length & $\mathrm{m}$ & $l_{\mathrm{b}}$ & 1.5 \\
\hline Hip to stand distance & $\mathrm{m}$ & $l_{\mathrm{b}, 1}$ & 1.4 \\
\hline Stand height & $\mathrm{m}$ & $l_{\mathrm{s}}$ & 1.4 \\
\hline Constraint boom mass & $\mathrm{kg}$ & $m_{\mathrm{b}}$ & 5.0 \\
\hline Cable mass & $\mathrm{kg}$ & $m_{\mathrm{c}}$ & 2.0 \\
\hline Counterbalance mass & $\mathrm{kg}$ & $m_{\mathrm{w}}$ & 0.0 \\
\hline Support electronics mass & $\mathrm{kg}$ & $m_{\mathrm{e}}$ & 20.0 \\
\hline
\end{tabular}

$$
I_{\mathrm{e}}=\frac{1}{12} m_{\mathrm{e}} l_{\mathrm{e}}^{2}
$$

This results in additional kinetic energy,

$$
K_{\mathrm{a}}=\frac{1}{2} I_{\mathrm{s}}\left(\dot{\phi}_{\mathrm{h}}^{2}+\dot{\phi}_{\mathrm{v}}^{2}\right)+\frac{1}{2} I_{\mathrm{e}} \dot{\phi}_{\mathrm{h}}^{2}
$$

where $\phi_{\mathrm{h}}$ and $\phi_{\mathrm{v}}$ are the horizontal and vertical angular displacements of RABBIT about the center stand (see Figure 6). The angles $\phi_{\mathrm{h}}$ and $\phi_{\mathrm{v}}$ may be approximated by

$$
\phi_{\mathrm{h}} \approx \frac{p_{\mathrm{H}}^{\mathrm{h}}(q)-p_{\mathrm{H}}^{\mathrm{h}}\left(q_{0}\right)}{l_{\mathrm{b}, 1}} \text { and } \phi_{\mathrm{v}} \approx \frac{p_{\mathrm{H}}^{\mathrm{v}}(q)-p_{\mathrm{H}}^{\mathrm{v}}\left(q_{0}\right)}{l_{\mathrm{b}, 1}}
$$

where $q_{0}$ is RABBIT's configuration at the beginning of a step and $p_{\mathrm{H}}^{\mathrm{h}}$ and $p_{\mathrm{H}}^{\mathrm{v}}$ are the horizontal and vertical positions of the hip.

There is also additional potential energy due to the boom, the counterbalance, and the cabling,

$$
\begin{aligned}
V_{\mathrm{a}}= & \underbrace{\frac{1}{2} g_{0} \frac{m_{\mathrm{b}}}{l_{\mathrm{b}}}\left(l_{\mathrm{b}, 1}^{2}-l_{\mathrm{b}, 2}^{2}\right) \sin \left(\phi_{\mathrm{v}}\right)}_{\text {boom }} \\
& \quad-\underbrace{g_{0} m_{\mathrm{w}} l_{\mathrm{b}, 2} \sin \left(\phi_{\mathrm{v}}\right)}_{\text {counterbalance }}+\underbrace{\frac{1}{2} g_{0} m_{\mathrm{c}} l_{\mathrm{b}, 1} \sin \left(\phi_{\mathrm{v}}\right)}_{\text {cabling }} .
\end{aligned}
$$

Note that the counterbalance mass may be chosen to negate the potential energy due to the boom and cabling. In the experiments described in Section 4, no counterbalance was used; the required counterbalance of $52 \mathrm{~kg}$ could not be securely fastened to the boom because of the short length of $l_{\mathrm{b}, 2}$.

The controllers used for the experiments reported in Section 4 were designed using equations of motion which included the boom dynamics. These equations of motion were calculated by first forming an updated Lagrangian - the planar model's Lagrangian with the kinetic energy $K_{\mathrm{a}}$ added and the potential energy $V_{\mathrm{a}}$ subtracted - and then using the method of Lagrange. Table 2 gives the parameter values for the boom system setup used for the experiments.

Aside from the ability to counterbalance the boom, the choice of boom length has other important considerations. The longer the boom, the better the approximation of RABBIT as a planar mechanical system; however, the longer the boom, the greater the dynamic effects of the additional kinetic (9) and potential (11) energies, and the greater the flexibility of the boom. Boom flexibility was found to be of great significance experimentally. The boom was initially chosen to be $3 \mathrm{~m}$ in length. Flexing of the tubular steel boom affected forces 
on RABBIT's hip large enough to cause foot slippage. In response, the boom was swapped for a $1.5 \mathrm{~m}$ boom, and the foot slippage problem subsided.

\subsubsection{Gear reducers and joint friction}

To allow smaller, lighter weight motors to be used, RABBIT has gear reducers between its motors and links. The gear reducers have two important effects on RABBIT's dynamics. The first effect is to add significant joint friction, which effectively eliminates all passive motions of the joints. The second effect is to approximately decouple the robot's dynamics, leaving the motor's rotor inertia as the only significant inertial load on the motor. Both effects were taken into consideration in the control implementation described in Section 3.4.

The joint friction was modeled by viscous and static friction terms,

$$
F(q, \dot{q}):=F_{\mathrm{v}} \dot{q}+F_{\mathrm{s}} \operatorname{sgn}(\dot{q})
$$

where $^{2} \quad F_{\mathrm{v}}=\operatorname{diag}\left(F_{\mathrm{v}, \mathrm{H}}, F_{\mathrm{v}, \mathrm{H}}, F_{\mathrm{v}, \mathrm{K}}, F_{\mathrm{v}, \mathrm{K}}\right)$ and $F_{\mathrm{S}}=$ $\operatorname{diag}\left(F_{\mathrm{s}, \mathrm{H}}, F_{\mathrm{s}, \mathrm{H}}, F_{\mathrm{s}, \mathrm{K}}, F_{\mathrm{s}, \mathrm{K}}\right)$. The identified values of RABBIT's frictional parameters are given in Table 1 . Note that both the viscous and static friction values are substantial; at the hip, the static friction is approximately ten percent of the motor/gear reducer system's peak available torque of $150 \mathrm{Nm}$.

Another, in some ways desirable, effect of gear reducers is to scale the inertial load seen by the motors. This scaling has the effect of approximately decoupling the robot's actuated dynamics so that the only significant dynamic terms are the inertia of the motors' rotors and the unactuated dynamics. Writing the model in motor coordinates makes this evident.

Define the motor shaft coordinates $\bar{q}:=N_{\mathrm{g}} q$ where $N_{\mathrm{g}}=$ $\operatorname{diag}\left(n_{\mathrm{g}}, n_{\mathrm{g}}, n_{\mathrm{g}}, n_{\mathrm{g}}, 1\right)$ and $n_{\mathrm{g}}$ are the gear reducers' gear ratio (the four gear reducers are identical). Since the torso, $q_{5}$, is unactuated, $\left(N_{\mathrm{g}}\right)_{55}=1$. When the motors' rotor inertias and the gear ratios are included in RABBIT's swing phase model, (1), and the model is written in the motor shaft coordinates, the equations of motion become

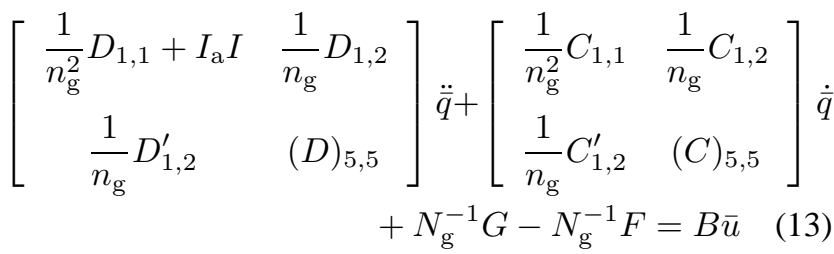

where $\bar{u}:=\left(\bar{u}_{1}, \bar{u}_{2}, \bar{u}_{3}, \bar{u}_{4}\right)$ are the torques supplied at the output shafts of the motors and $I_{\mathrm{a}}$ is the inertia of the motors' rotors (the four motors are identical). The result is that the actuated dynamics are approximately decoupled and the block of actuated dynamics is approximately decoupled from the unactuated dynamics. RABBIT's motors' rotor inertia and gear ratio are given in Table 1.

\footnotetext{
${ }^{2}$ As is commonly done to circumvent the difficulties associated with the discontinuity of the signum function, in implementation, a scaled arctangent function was used in its place, i.e., for large $\tau$,

$$
\operatorname{sgn}(x) \approx \frac{2}{\pi} \tan ^{-1}(\tau x) .
$$
}

\subsubsection{The walking surface}

The floor on which RABBIT walks is concrete with $30 \mathrm{~cm}$ wide cabling access trenches covered with $4 \mathrm{~mm}$ steel plates. In preliminary experiments it was found that after stepping on one of the four plates crossing RABBIT's path, RABBIT would slow significantly. Since the gait-change in the shape over a step-was the same (see Section 3.1), this indicated that the energy dissipation due to impacting the concrete surface is less than the energy dissipation due to impacting the steel plates. To help make the walking surface uniform, the floor was covered with $1.5 \mathrm{~cm}$ particle board, which was then covered with a layer of $3 \mathrm{~mm}$ rubber (see Figure 3). Aside from helping to make the walking surface uniform, the rubber layer was added in hopes of extending the life of RABBIT by providing a modest amount of compliance.

\section{Controlling dynamically stable walking}

The approach to the control of walking taken here is to encode the task of walking as a set of holonomic constraints on the robot's actuated DOF parameterized by the robot's unactuated DOF. With this approach, the closed-loop system is rendered autonomous (time-invariant) and formal analysis of the stability properties of the resulting gait is possible [12]. Beyond analysis, in [33], [34] a framework using this approach was developed for systematic design and performance enhancement of controllers that induce walking with $a$ priori known kinematic and dynamic properties. This section provides a succinct summary of that work and interprets the use of holonomic constraints as imposing virtual constraintsholonomic constraints imposed via feedback. "Virtual constraint" is used to differentiate these constraints from physical constraints that are imposed via, for example, a geared mechanism or a cam and link system.

\subsection{Virtual constraints and hybrid zero dynamics}

Since the model for walking is underactuated-due to no ankle torque - the dynamics compatible with the constraints being exactly imposed are nontrivial. During the single support phase these dynamics correspond to the inverted pendulumlike dynamics of the center of mass about the stance leg end. A means for calculating these dynamics - called the zero dynamics-via an output design problem is given in [34]. It is shown that the zero dynamics can be designed to be an invariant sub-dynamic of the full hybrid model in spite of the discontinuous dynamics introduced by impacts. In this case, existence of a limit cycle of the zero dynamics is easily determined. This is interesting because exponentially stable periodic orbits of the hybrid zero dynamics are exponentially stabilizable periodic orbits of (5).

The main results of [34] are now summarized. Consider the model (2) with the following output function

$$
y=h(x):=h_{0}(q)-h_{d} \circ \theta(q)
$$

where $h_{0}(q)$ specifies 4 independent quantities that are to be controlled and $h_{d} \circ \theta(q)$ specifies the desired evolution of these quantities as a function of the scalar, monotonic quantity 


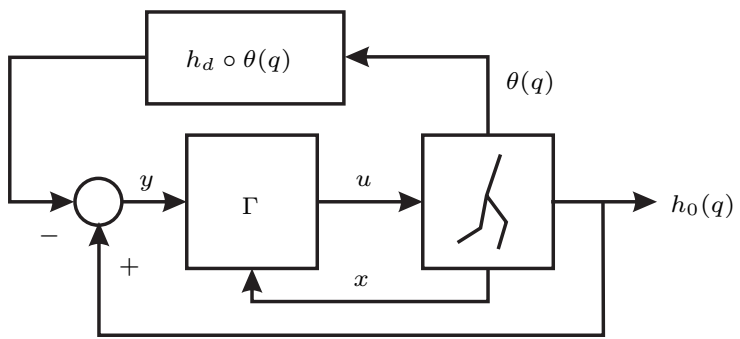

Fig. 7. Block diagram of a time invariant controller. The controller $\Gamma$ forces the signal $y=h_{0}(q)-h_{d} \circ \theta(q)$ to zero so that the signal $h_{0}(q)$ tracks the function $h_{d} \circ \theta(q)$.

$\theta(q)$. Driving $y$ to zero will force $h_{0}(q)$ to track $h_{d} \circ \theta(q)$; see Figure 7. Intuitively, the posture of the robot is being controlled by virtual constraints-here a holonomic constraint parameterized by $\theta(q)$. Unlike physical constraints, virtual constraints may be reconfigured electronically via change in feedback law, whereas reconfiguration of physical constraints requires mechanism redesign; the next section discusses their difference in detail. Note that the control of walking using virtual constraints is not a trajectory tracking scheme since the desired evolution of $h_{0}(q)$ is slaved to a function of $\theta(q)$ and not time. For this reason, the resulting closed-loop system is autonomous.

Choosing

$$
\begin{aligned}
h_{0}(q) & :=H_{0} q \\
\theta(q) & :=c q
\end{aligned}
$$

where $H_{0} \in \mathbb{R}^{5 \times 4}, c \in \mathbb{R}^{5 \times 1}$ allows hypotheses HH1)-HH4) to be easily satisfied, which guarantees the existence of the swing phase zero dynamics,

$$
\dot{z}=f_{\text {zero }}(z),
$$

which evolves on the two-dimensional embedded sub-manifold

$$
Z:=\left\{x \in T \tilde{\mathcal{Q}} \mid h(x)=0, L_{f} h(x)=0\right\}
$$

where $\tilde{\mathcal{Q}} \subset \mathcal{Q}$ is the set where ${ }^{3} L_{g} L_{f} h(x)$, the decoupling matrix, is invertible. The feedback control

$$
u^{*}(x)=-\left(L_{g} L_{f} h(x)\right)^{-1} L_{f}^{2} h(x)
$$

${ }^{3}$ (This definition of the Lie derivative is taken from [16, pp. 496-496].) Let $f$ be a smooth vector field, $N$ a smooth manifold of dimension $n$, and $\lambda$ a smooth real-valued function on $N$. The (Lie) derivative of $\lambda$ along $f$ is a function $N \rightarrow \mathbb{R}$, written $L_{f} \lambda$ and

$$
\left(L_{f} \lambda\right)(p):=(f(p))(\lambda)
$$

(i.e., $\left(L_{f} \lambda\right)(p)$ is the value at $\lambda$ of the tangent vector $f(p)$ at $p$ ). In local coordinates, $L_{f} \lambda$ is represented by

$$
\left(L_{f} \lambda\right)\left(x_{1}, \ldots, x_{n}\right)=\left(\frac{\partial \lambda}{\partial x_{1}} \ldots \frac{\partial \lambda}{\partial x_{n}}\right)\left(\begin{array}{c}
f_{1} \\
\vdots \\
f_{n}
\end{array}\right) .
$$

If $f_{1}, f_{2}$ are vector fields and $\lambda$ is a real-valued function, then

$$
L_{f_{1}} L_{f_{2}} \lambda:=L_{f_{1}}\left(L_{f_{2}} \lambda\right)
$$

and

$$
L_{f_{1}}^{2} \lambda:=L_{f_{1}} L_{f_{1}} \lambda .
$$

renders $Z$ invariant under the swing dynamics; that is, for every $z \in Z, f_{\text {zero }}(z):=f(z)+g(z) u^{*}(z) \in T_{z} Z$. When expressed in coordinates $\left(\xi_{1}, \xi_{2}\right)=(\theta(q), \gamma(q, \dot{q}))$ where $\gamma(q, \dot{q}):=\gamma_{0}(q) \dot{q}$ and $\gamma_{0}(q)$ is the last row of $D$, the massinertia matrix, the zero dynamics (17) take the form [34, Thm. 1]

$$
\begin{aligned}
& \dot{\xi}_{1}=\kappa_{1}\left(\xi_{1}\right) \xi_{2} \\
& \dot{\xi}_{2}=\kappa_{2}\left(\xi_{1}\right) .
\end{aligned}
$$

If $S \cap Z$ is a smooth, one-dimensional, embedded sub-manifold of $T \mathcal{Q}$, and if $\Delta(S \cap Z) \subset Z$, then the nonlinear system with impulse effects,

$$
\begin{array}{lll}
\dot{z}=f_{\text {zero }}(z) & z^{-} \notin S \cap Z \\
z^{+}=\Delta\left(z^{-}\right) & z^{-} \in S \cap Z,
\end{array}
$$

with $z \in Z$, is an invariant sub-dynamic of the model (5) and is called the hybrid zero dynamics of (5). In the coordinates $\left(\zeta_{1}, \zeta_{2}\right)=\left(\theta, \frac{1}{2} \gamma^{2}\right)$, the Poincaré return map of the hybrid zero dynamics, $\rho: S \cap Z \rightarrow S \cap Z$, is given by

$$
\rho\left(\zeta_{2}^{-}\right)=\delta_{\text {zero }}^{2} \zeta_{2}^{-}-V_{\text {zero }}\left(\theta^{-}\right),
$$

with domain of definition

$$
\left\{\zeta_{2}^{-}>0 \mid \delta_{\text {zero }}^{2} \zeta_{2}^{-}-V_{\text {zero }}^{\mathrm{MAX}} \geq 0\right\}
$$

where $\delta_{\text {zero }}, V_{\text {zero }}$, and $V_{\text {zero }}^{\mathrm{MAX}}$ are constants calculated from (22), and $\theta^{-}:=\theta\left(q^{-}\right), q^{-} \in S \cap Z$. If $\delta_{\text {zero }}^{2} \neq 1$ and

$$
\zeta_{2}^{*}:=-\frac{V_{\mathrm{zero}}\left(\theta^{-}\right)}{1-\delta_{\mathrm{zero}}^{2}}
$$

is in the domain of definition of $\rho$, then it is the fixed point of $\rho$ [34, Thm. 3]. Moreover, if $\zeta_{2}^{*}$ is a fixed point, then $\zeta_{2}^{*}$ is an exponentially stable equilibrium point of

$$
\zeta_{2}(k+1)=\rho\left(\zeta_{2}(k)\right)
$$

if, and only if, $0<\delta_{\text {zero }}^{2}<1$, and in this case, its domain of attraction is (24), the entire domain of definition of $\rho$. Therefore, the hybrid zero dynamics admit a non-trivial, exponentially stable periodic orbit if, and only if, the following two inequality constraints hold [34, Cor. 1]

$$
0<\delta_{\text {zero }}^{2}<1
$$

and

$$
\frac{\delta_{\text {zero }}^{2}}{1-\delta_{\text {zero }}^{2}} V_{\text {zero }}\left(\theta^{-}\right)+V_{\text {zero }}^{\mathrm{MAx}}<0 .
$$

Exponentially stable periodic orbits of the hybrid zero dynamics are exponentially stabilizable periodic orbits of (5), [34, Sec. IV.C].

Specialization of $h_{d}$ to a vector of Bézier polynomials makes achieving the invariance condition, $\Delta(S \cap Z) \subset Z$, simple as well as providing a finite parameterization of the zero dynamics. Finite parameterization allows $h(x)$-and thus the hybrid zero dynamics - to be tuned using standard parameter optimization techniques [34, Sec. VI]. 


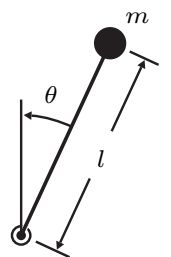

Fig. 8. A horizontal, variable length pendulum used to explain virtual constraints.

\subsection{Virtual versus physical constraints}

Though virtual constraints and physical constraints impose the same kinematic behavior, the resulting dynamic behavior is different. To illustrate this difference, consider the horizontal variable length pendulum depicted in Figure 8. The distance from the point mass $m$ to the rotation point is $l$ and may vary. Since the pendulum is horizontal, its Lagrangian is equal to the kinetic energy,

$$
L=K=\frac{1}{2} m\left(\dot{l}^{2}+l^{2} \dot{\theta}^{2}\right) .
$$

Two different scenarios will be considered. First, the length, $l$, will be constrained to be a function of $\theta$ via a physical constraint. Second, $l$ will be constrained via a virtual constraint.

In the first case, suppose that a physical constraint is designed such that $l=l_{d}(\theta)$. Then, the equation of motion is

$$
\begin{aligned}
& m\left(\left(\frac{\partial l_{d}(\theta)}{\partial \theta}\right)^{2}+\left(l_{d}(\theta)\right)^{2}\right) \ddot{\theta} \\
& \quad+m \frac{\partial l_{d}(\theta)}{\partial \theta}\left(\frac{\partial^{2} l_{d}(\theta)}{\partial \theta^{2}}+l_{d}(\theta)\right) \dot{\theta}^{2}=0 .
\end{aligned}
$$

Now suppose that $l$ is constrained via a virtual constraint. In this case, the length $l$ is treated as a controlled quantity, and the equations of motion may be calculated from the Lagrangian (29) to be

$$
\begin{aligned}
\ddot{\theta} & =-\frac{2}{l} \dot{l} \dot{\theta} \\
\ddot{l} & =l \dot{\theta}^{2}+\frac{1}{m} u
\end{aligned}
$$

where $u$ is an input used to control the length $l$. To do so, an output on the system (31) is formed as

$$
y=l-l_{d}(\theta)
$$

such that $y \equiv 0$ implies $l \equiv l_{d}(\theta)$. As long as $\partial l_{d}(\theta) / \partial \theta \neq$ 0 , the output (32) is of relative degree two [16]. Hence, differentiating twice yields

$$
\ddot{y}=l \dot{\theta}^{2}-\frac{\partial l_{d}^{2}(\theta)}{\partial \theta^{2}} \dot{\theta}^{2}+\frac{2}{l} \frac{\partial l_{d}(\theta)}{\partial \theta} \dot{i} \dot{\theta}+\frac{1}{m} u
$$

The output dynamics (33) may be stabilized with

$$
u=m\left(-l \dot{\theta}^{2}+\frac{\partial l_{d}^{2}(\theta)}{\partial \theta^{2}} \dot{\theta}^{2}-\frac{2}{l} \frac{\partial l_{d}(\theta)}{\partial \theta} i \dot{\theta}-K_{D} \dot{y}-K_{P} y\right)
$$

for $K_{D}, K_{P}>0$. Under the constraint $l \equiv l_{d}(\theta)$, the system state must evolve on the $\operatorname{set}^{4} Z:=\left\{(\theta, \dot{\theta}, l, \dot{l}) \in \mathrm{S} \times \mathbb{R}^{3} \mid l-\right.$

\footnotetext{
${ }^{4}$ Here $\mathrm{S}$ is the topological space the unit circle, not the walking surface see Section 2.1.3.
}

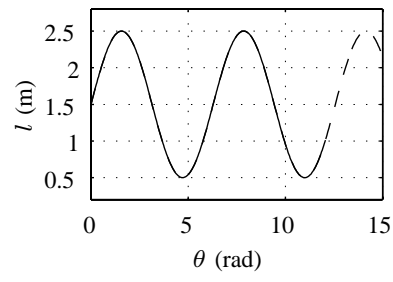

(a) Kinematic behavior

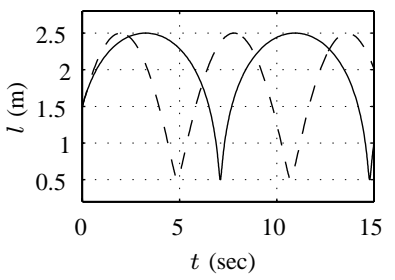

(b) Dynamic behavior
Fig. 9. Kinematic and dynamic behaviors of the horizontal pendulum. The dashed lines correspond to the constraint $l=\sin (\theta)+1.5$ imposed via a physical constraint, whereas the solid corresponds to the same constraint imposed via a virtual constraint.

$\left.l_{d}(\theta)=0\right\}$. The maximal internal dynamics compatible with the output being identically zero, the zero dynamics, are

$$
m\left(l_{d}(\theta)\right)^{2} \ddot{\theta}+2 m \frac{\partial l_{d}(\theta)}{\partial \theta} l_{d}(\theta) \dot{\theta}^{2}=0
$$

and the unique control required to enforce $l \equiv l_{d}(\theta)$, $u^{*}$, is readily calculated from (34).

While the kinematic behavior of the zero dynamics resulting from the virtual constraint $l=l_{d}(\theta)$ is identical to that resulting from the physical constraint $l=l_{d}(\theta)$, their dynamic behavior is different, cf. (30) and (35). Figure 9 illustrates this difference for the constraint $l_{d}=\sin (\theta)+1.5$. For this example, $m=1$ and the system (30) was initialized with $(\theta, \dot{\theta})=(0,1)$ and the system (35) was initialized with $(\theta, \dot{\theta}, l, \dot{l})=(0,1,1.5,1) \in Z$. The difference between these two realizations lies in $u^{*}$, the energy entering the system in the virtual constraint realization which does not appear in the physical constraint realization.

\subsection{Interpreting the (swing phase) zero dynamics}

Much in the way that it has been proposed that the spring loaded inverted pendulum is a template for running [26], [29], it has been proposed, though less formally, that an inverted pendulum is the template for walking [7], [17]-[19], [22]. From Figure 1 it might seem that the dynamics that result from imposing virtual constraints - the swing phase zero dynamics, (20) and (21) — should be the dynamics of a length and inertia varying inverted pendulum, i.e., the length, $l$, and the inertia about the center of mass (COM), $J$, vary as function of $\xi_{1}$. If this were true it would suggest this pendulum as a new control template (or target) in the design of future controllers. Unfortunately, it is not true.

Using the angular momentum balance theorem [27], the rate of change of the angular momentum of the robot about the stance leg end during the swing phase, $\dot{\xi}_{2}$, is equal to the external applied torque,

$$
\dot{\xi}_{2}=g_{0} M p_{\mathrm{COM}}^{\mathrm{h}}
$$

where $g_{0}$ is the acceleration due to gravity, $M$ is the robot's mass and $p_{\mathrm{COM}}^{\mathrm{h}}$ is the horizontal position of the robot's center of mass; see Figure 1. Suppose $\xi_{1}$ is defined as in Figure 1. 
Then, for output (14), if $y \equiv 0$, then $p_{\mathrm{COM}}^{\mathrm{h}}=p_{\mathrm{COM}}^{\mathrm{h}}\left(\xi_{1}\right)$ and $l=l\left(\xi_{1}\right)$ so that

$$
\kappa_{2}\left(\xi_{1}\right)=g_{0} M l\left(\xi_{1}\right) \sin \left(\xi_{1}\right) .
$$

Expressing (20) as $\xi_{2}=I_{\text {zero }}\left(\xi_{1}\right) \dot{\xi}_{1}$, where ${ }^{5} I_{\text {zero }}\left(\xi_{1}\right)=$ $1 / \kappa_{1}\left(\xi_{1}\right)$ is an inertial term, allows the zero dynamics (20) and (21) to be written as a second order system,

$$
I_{\text {zero }}\left(\xi_{1}\right) \ddot{\xi}_{1}+\frac{\partial I_{\text {zero }}\left(\xi_{1}\right)}{\partial \xi_{1}}\left(\dot{\xi}_{1}\right)^{2}-g_{0} M l\left(\xi_{1}\right) \sin \left(\xi_{1}\right)=0 .
$$

The equation of motion for a length and inertia varying pendulum can be easily derived using the method of Lagrange. The kinetic energy is $K\left(\xi_{1}\right)=1 / 2 I\left(\xi_{1}\right) \dot{\xi}_{1}^{2}$ where

$$
I\left(\xi_{1}\right)=M\left(\frac{\partial l\left(\xi_{1}\right)}{\partial \xi_{1}}\right)+M\left(l\left(\xi_{1}\right)\right)^{2}+J\left(\xi_{1}\right) .
$$

The potential energy is $V\left(\xi_{1}\right)=M g_{0} l\left(\xi_{1}\right) \cos \left(\xi_{1}\right)$, and, hence, the equation of motion ${ }^{6}$ is,

$$
\begin{aligned}
I\left(\xi_{1}\right) \ddot{\xi}_{1} & +\frac{1}{2} \frac{\partial I\left(\xi_{1}\right)}{\partial \xi_{1}} \dot{\xi}_{1}^{2} \\
& +M g_{0}\left(\frac{\partial l\left(\xi_{1}\right)}{\partial \xi_{1}} \cos \left(\xi_{1}\right)-l\left(\xi_{1}\right) \sin \left(\xi_{1}\right)\right)=0 .
\end{aligned}
$$

Comparing the swing phase zero dynamics (38) and the dynamics for the length and inertia varying pendulum (40), it is evident that what is suggested by Figure 1 does not hold, which also implies that the swing phase zero dynamics are not those of an inverted pendulum, or an inverted length and inertia varying pendulum. It is interesting to note, however, that if the length and inertia varying inverted pendulum had a torque, $u$, acting between the pendulum and ground, i.e.,

$$
\begin{aligned}
I\left(\xi_{1}\right) \ddot{\xi}_{1} & +\frac{1}{2} \frac{\partial I\left(\xi_{1}\right)}{\partial \xi_{1}} \dot{\xi}_{1}^{2} \\
& +M g_{0}\left(\frac{\partial l\left(\xi_{1}\right)}{\partial \xi_{1}} \cos \left(\xi_{1}\right)-l\left(\xi_{1}\right) \sin \left(\xi_{1}\right)\right)=u,
\end{aligned}
$$

where

$$
u=-\frac{1}{2} \frac{\partial I\left(\xi_{1}\right)}{\partial \xi_{1}} \dot{\xi}_{1}^{2}+M g_{0} \frac{\partial l\left(\xi_{1}\right)}{\partial \xi_{1}} \cos \left(\xi_{1}\right),
$$

then, the forms of (38) and (41) with $u$ as in (42) would be identical. $^{7}$ Matching the inertial terms, $I$ and $I_{\text {zero }}$, however, does not yield a positive definite $J$. That is, supposing $I_{\text {zero }}$ has the form of $I$ given in (39) implies $J\left(\xi_{1}\right)=I_{\text {zero }}-$ $M\left(\partial l\left(\xi_{1}\right) / \partial \xi_{1}\right)-M\left(l\left(\xi_{1}\right)\right)^{2}$ where $l$ is the distance from the stance leg end to the COM. For every example worked by the authors, $J$ is sign indefinite.

\footnotetext{
${ }^{5}$ The proposition [34, Prop. 1] ensures that $\kappa_{1}\left(\xi_{1}\right)$ is never zero whenever the robot successfully completes a step.

${ }^{6}$ If $l$ and $J$ do not vary as a function of $\xi_{1}$, then $I\left(\xi_{1}\right)=I, l\left(\xi_{1}\right)=l$ and (40) reduces to the equation of motion for an inverted pendulum, $I \ddot{\xi}_{1}-$ $M g_{0} l \sin \left(\xi_{1}\right)=0$.

${ }^{7}$ The justification for this input is to account for the energy entering the robot's dynamics via the control $u^{*}$ given in (19).
}

\subsection{The control algorithm implementation: imposing the virtual constraints}

The swing phase zero dynamics (20) and (21) are independent of the feedback used to zero the output that gives rise to them. The feedback introduced in [34, Sect. IV-C], a computed torque pre-feedback plus finite time converging controllers, is one possible feedback. The input-output linearization of the computed torque pre-feedback decoupled the dynamics resulting in a chain of four double integrators. The finitetime converging controllers stabilized the origin of the double integrators in finite time, enabling the stability of the robot's walking motion to be assessed via the hybrid zero dynamics' scalar return map. In light of the decoupling effect of the reducers (see Section 2.2.2) and the likely inaccuracy of the parameter identification, high-gain decoupled PD controllers were used in place of the feedback given in [34, Sect. IV-C] to impose the virtual constraints on RABBIT. It was found that this control was able to zero the outputs sufficiently well to induce walking with dynamic characteristics similar to the theoretical design.

For the experiments described in this paper, outputs of the form (14) with $h_{0}(q)$ and $\theta(q)$ as in (15) and (16) were used with

$$
\begin{aligned}
H_{0} & =\left[\begin{array}{ll}
I & 0
\end{array}\right] \\
c & =\left[\begin{array}{lllll}
-1 & 0 & -1 / 2 & 0 & -1
\end{array}\right]
\end{aligned}
$$

which results in the output

$$
y=\left(q_{1}, q_{2}, q_{3}, q_{4}\right)^{\prime}-h_{d} \circ \theta(q) .
$$

Figure 4(b) gives $\theta(q)$ corresponding to this choice of $c$. The Bézier polynomial order, $M$, was chosen to be 6 , which left five free parameters to be chosen for each output (two parameters per output are used to impose invariance; see [34, Rem. 4]). This implied a total of 20 output function parameters to be chosen via optimization. The optimization problem was posed as described in [34, Sec. VI] to choose the 20 free parameters of $\alpha$ by approximately minimizing the cost

$$
J(\alpha):=\frac{1}{p_{2}^{\mathrm{h}}\left(q^{-}\right)} \int_{0}^{T_{I}\left(\xi_{2}^{-}\right)}\left\|u^{*}(t)\right\|_{2}^{2} d t
$$

where $q^{-} \in S \cap Z, T_{I}\left(\xi_{2}^{-}\right)$corresponds to the step duration, $p_{2}^{\mathrm{h}}\left(q_{0}^{-}\right)$corresponds to step length, and $u^{*}(t)$ is the result of evaluating (19) along a solution of the hybrid zero dynamics. The hybrid zero dynamics used in the optimization process were updated to accommodate the effects of the additional dynamics caused by the inertia of the boom system and friction of the gear reducers. The effect of the non-rigidity of the walking surface was also important to accommodate.

The tradeoff between the dissipation due to impacts and the energy gained through shape change (cf. [34, Thm. 3]) determine the closed-loop system's average walking rate and stability. Imperfections in the model parameters and unmodeled dynamics during the swing phase affect the energy gained through shape change. Imperfections in the impact model affect the amount of energy dissipated. To study the latter, RABBIT was simulated using a compliant ground contact 


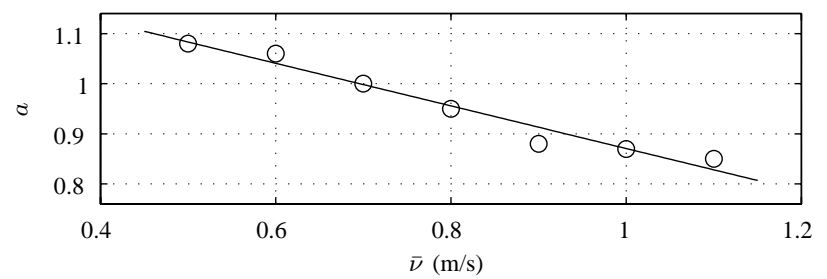

Fig. 10. Average walking rate verses impact map scaling constant $a$. The solid line is a least squares fit to empirically determined impact scalings (indicated by circles). This apparently linear relationship between average walking rate and impact scaling is reminiscent of the classical coefficient of restitution relation, $e=1-a v_{0}$, where $e$ is the coefficient of restitution, $a$ is some material-dependent constant, and $v_{0}$ is the impacting velocity [8, p. 258]. It is hypothesized that this approximately linear relation will hold for other walking surfaces, suggesting it as a means of identifying the surface to determine how the rigid impact model, i.e., $\delta_{\text {zero }}$, should be modulated as a function of $\bar{\nu}$.

Table 3. Experiment control parameter values.

\begin{tabular}{c|c|c|c}
\hline Control parameter & Units & Label & Value \\
\hline \hline \multirow{2}{*}{ Proportional gains } & \multirow{2}{*}{$\mathrm{N}$} & $K_{P, \mathrm{H}}$ & 2000 \\
\cline { 3 - 4 } & & $K_{P, \mathrm{~K}}$ & 1500 \\
\hline \multirow{2}{*}{ Derivative gains } & \multirow{2}{*}{$\mathrm{Ns}$} & $K_{D, \mathrm{H}}$ & 10 \\
\cline { 3 - 4 } & & $K_{D, \mathrm{~K}}$ & 10 \\
\hline
\end{tabular}

model described in [23]. It was found that stability was preserved, but the steady-state average walking rate differed from the average walking rate designed assuming rigid impacts. This was also found experimentally.

For the wood and rubber walking surface, it was found that in the design of walking motions, the amount of energy dissipated at impact had to be scaled to be less than the rigid model predicted at low walking speeds and more at higher walking speeds. This was accomplished through trial and error by scaling $\delta_{\text {zero }}$ (see [34, Sec. IV-A] for its definition) by some constant $a$. A series of controllers over a range of values of $a$ were generated and then evaluated using the experiment procedure described in Section 4 to determine their steady-state average walking rates. The value of $a$ resulting in a controller that induced the desired average walking rate was recorded. Figure 10 gives a plot of these values of $a$ verses the corresponding average walking rate. Surprisingly, the relationship is approximately linear; the least squares fit is $a(\bar{\nu})=1.2957-0.4250 \bar{\nu}$.

To zero the output resulting from optimization on the hybrid zero dynamics updated to accommodate the implementation issues, the decoupled, PD controller with friction compensation $^{8}$

$$
u=-K_{P} e-K_{D} \dot{e}+F_{\mathrm{v}} h_{d} \circ \hat{\theta}(\hat{q})+F_{\mathrm{s}} \operatorname{sgn}(e)
$$

was used where the terms $F_{\mathrm{v}} h_{d} \circ \hat{\theta}(q)$ and $F_{\mathrm{s}} \operatorname{sgn}(e)$ correspond to feed-forward viscous and static friction compensation terms and $K_{P}=\operatorname{diag}\left(K_{P, \mathrm{H}}, K_{P, \mathrm{H}}, K_{P, \mathrm{~K}}, K_{P, \mathrm{~K}}\right)$ and $K_{D}=\operatorname{diag}\left(K_{D, \mathrm{H}}, K_{D, \mathrm{H}}, K_{D, \mathrm{~K}}, K_{D, \mathrm{~K}}\right)$ are the proportional and derivative gains given in Table 3 . The error signals are

\footnotetext{
${ }^{8}$ The friction compensation terms are due to C. Canudas-de-Wit.
}

defined as

$$
e:=H_{0} \hat{q}-h_{d} \circ \hat{\theta}(\hat{q}) \text { and } \dot{e}:=H_{0} \dot{\hat{q}}-\frac{\partial h_{d}}{\partial \theta} \dot{\hat{\theta}}(\dot{\hat{q}})
$$

where $(\hat{q}, \dot{\hat{q}})$ is RABBIT's state with relabeling,

$$
(\hat{q}, \dot{\hat{q}}):= \begin{cases}(q, \dot{q}) & \text { if stance leg is right leg } \\ \left(\Delta_{q} q, \Delta_{q} \dot{q}\right) & \text { if stance leg is left leg. }\end{cases}
$$

A simple state machine was used to determine the current stance leg as required by (49). Since $h_{d}$ is only designed for ${ }^{9}$ $0 \leq\left(\theta(q)-\theta^{+}\right) /\left(\theta^{-}-\theta^{+}\right) \leq 1$, where $\theta^{-}:=\theta\left(q^{-}\right)$and $\theta^{+}:=\theta \circ \Delta_{q}\left(q^{-}\right), q^{-} \in S \cap Z$, the scalar function of the robot's states $\theta(q)$ was saturated,

$$
(\hat{\theta}(q), \dot{\hat{\theta}}(q)):= \begin{cases}(\theta(q), \dot{\theta}(\dot{q})) & 0<\frac{\theta(q)-\theta^{+}}{\theta--\theta^{+}}<1 \\ \left(\theta^{-}, 0\right) & \frac{\theta(q)-\theta^{+}}{\theta^{-}-\theta^{+}}>1 \\ \left(\theta^{+}, 0\right) & \frac{\theta(q)-\theta^{+}}{\theta^{-}-\theta^{+}}<0 .\end{cases}
$$

The velocities were estimated using a five-point numerical differentiator described in [5] applied to the encoder outputs.

The PD based feedback (47) was chosen over a sliding mode, or finite-time converging controller because of its robustness to noise and uncertainty. The feedback (47) provided surprisingly good joint-level tracking (see, for example, Figure 12).

The feedback (47)-(50) was implemented on the dSPACE DS1103 system running with a sample period of $1.5 \mathrm{~ms}$ $(667 \mathrm{~Hz})$. To provide high-level control, the feedback implementation has a state machine which provides an interface between the user and the lower-level, continuous control. The implementation also has safety mechanisms which set the commanded control signals to zero in the event of an anomalous condition, such as a joint exceeding a position limit, or upon user request.

\section{Experiments}

This section describes six experiments which highlight the capabilities and robustness of controllers designed via the theoretical framework summarized in Section 3 and the additional tools presented in [33] and Appendix 2. The experiments reported were performed during a three week research visit lasting from February 24th until March 14th 2003. At the start of the visit, RABBIT had just been installed into its permanent location, pictured in Figure 3, where about 200 consecutive steps (6 to 7 laps about the center stand) are possible; the limit on the total number of steps is due to the winding of the power and Ethernet cabling about the center stand. During the visit, many small details had to be addressed to improve the experimental platform before the reported experiments could be performed.

The experiments were conducted as follows: the experiment began with the robot suspended in the air, lifted by an experimenter. After an encoder calibration phase, the robot was servoed to a configuration $(q, \dot{q})=\left(q_{0}, 0\right) \in \Delta\left(S \cap Z_{\alpha}\right)$

\footnotetext{
${ }^{9}$ The parameterization of Bézier polynomials is such that they are only
} defined on the unit interval. 


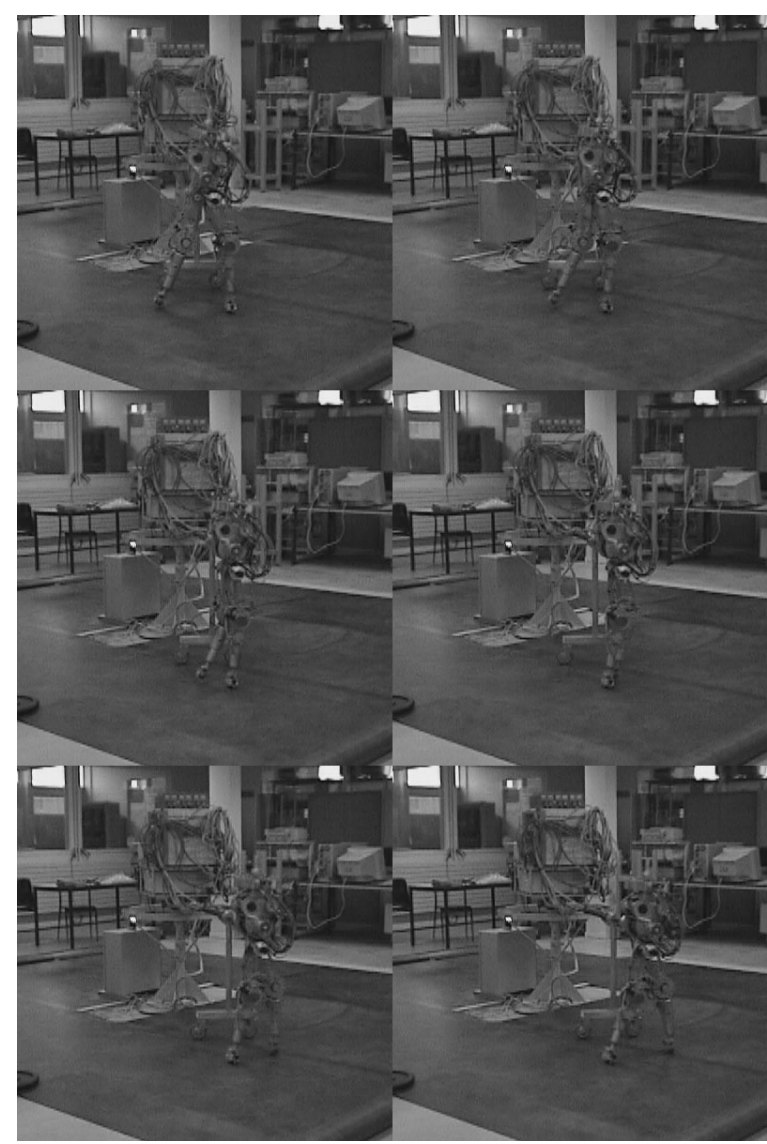

Fig. 11. Video frames of RABBIT taking a step. The interval between frames is $120 \mathrm{~ms}$. Note the "training wheels" attached to the boom to provide a measure of safety.

and then placed on the ground. Once on the ground, data collection was initiated and the control was switched to the virtual constraint based feedback controller (47)-(50). This was possible because $(q, \dot{q})=\left(q_{0}, 0\right) \in \Delta\left(S \cap Z_{\alpha}\right)$ is an asymptotically stable point under the hybrid zero dynamics based controller. To initiate walking, an experimenter pushed the robot's torso-temporarily fully actuating the robot's underactuated dynamics - supplying the energy required to land the robot's state in the basin of attraction of the Poincare return map. Once in motion, the robot reached steady state walking within ten to twenty steps. To stop the robot, an experimenter grabbed the torso-again, fully actuating the robot's dynamics - slowing the robot to a stop in double support. This ability to interact with the robot's dynamics by pushing on the robot is a consequence of the zero dynamics' parameterization by $\theta(q)$. Through mechanical coupling, forces on the robot drive $\theta(q)$ which, in turn, determine the evolution of the robot's actuated DOF. Figure 11 gives video frames of RABBIT taking a step for a typical walking motion. Videos of these experiments are available at [31].

\subsection{Walking at a fixed rate}

These first two experiments illustrate the performance of controllers designed via the theoretical framework summarized in Section 3.

\subsubsection{Walking at $0.7 \mathrm{~m} / \mathrm{s}$}

In this first experiment, RABBIT was controlled with a feedback designed to induce walking at $0.7 \mathrm{~m} / \mathrm{s}$. The experiment lasted approximately 93 seconds during which RABBIT took 170 steps. Figures 12-17 are plots of various quantities of interest over a representative time interval containing approximately five steps. Figures 12 and 13 give the tracking performance. Figure 15 gives the commanded control signals. The peak commanded torque is less than $100 \mathrm{Nm}$, two thirds of the actuators' $150 \mathrm{Nm}$ maximum. Figure 14 gives the trace of the torso angle. Note the torso angle is not a directly controlled quantity. Figure 16 gives the trace of the horizontal angular displacement of the boom, $\phi_{\mathrm{h}}$. As indicated by Figure 16, the robot took approximately six laps about the center stand to complete the 170 steps. Note the constant slope and monotonicity of the trace of $\phi_{\mathrm{h}}$ indicating smooth, horizontal motion of the hip. Figure 17 gives the step length, step duration, and the ratio of step length to step duration, step rate. The data points of Figure 17 were calculated upon swing foot touchdown declaration. The step lengths given in Figure 17 were calculated using the measured joint angles and the robot's identified link lengths. In each plot of Figure 17, squares indicate data points corresponding to the inner legthe leg closer to the center stand-and circles indicate data points corresponding to the outer leg-the leg further from the center stand. Labeling the data points in this way reveals differences between the inner and outer legs in step length, step duration, and step rate. Since the two legs are, to within close approximation, identical, the difference is likely due to the non-sagittal plane dynamics created by the boom system. Aside from the differences between the inner and outer legs, the variances in step length and step duration have several contributing factors. The ones believed to be most significant are nonuniformity in the walking surface, variance in the declaration of leg touchdown, and flexibility in the robot's joints. Careful comparison of Figure 17 with Figure 16 reveals that the step rate is periodic in $\phi_{\mathrm{h}}$. The periodic change with $\phi_{\mathrm{h}}$ is due to nonuniformity in the walking surface: one section of the wood and rubber walking surface was not firmly lying on the ground because of unevenness in the underlying concrete floor.

\subsubsection{Demonstration of robustness to perturbations}

This second experiment demonstrates the robustness of controllers designed via the theoretical framework. Two types of perturbations were applied to RABBIT controlled by a feedback designed to induce walking at $0.9 \mathrm{~m} / \mathrm{s}$. The first was a $10 \mathrm{~kg}$ mass added to the torso, which resulted in a shift of the average walking rate from $0.9 \mathrm{~m} / \mathrm{s}$ to $1.0 \mathrm{~m} / \mathrm{s}$. (In the fifth experiment, described in Section 4.2.3, it will be demonstrated that the designed fixed average walking rate may be recovered through the use of event-based integral control.) In addition to the sizable perturbation to the robot's model (the robot's nominal weight is $32 \mathrm{~kg}$ ), the second perturbation was aperiodic, short duration forces applied to the RABBIT's torso by an experimenter in both the forward and reverse directions. Despite both these significant perturbations, RABBIT did not 
fall during the experiment which lasted approximately 74 seconds where RABBIT took 164 steps.

Figures 18 and 19 are plots of the actual and desired joint trajectories and the commanded control signals over a representative time interval in which the robot was pushed in the forward direction (at approximately 20.5 seconds) and in the reverse direction (at approximately 29 seconds). Note that the change in the reference motion, $h_{d}$, during the application of these forces. The commanded control signals are within the actuators' limits, except during the force perturbations when they saturate (see Figure 19).

\subsection{Transitioning and event-based within-stride control}

The four experiments below illustrate the transitioning controller developed in [33] and the event-based controller developed in Appendix 2. Both techniques exploit freedom in the output function parameter choice while respecting invariance of the associated zero dynamics manifold.

\subsubsection{Transitioning between controllers}

This third experiment demonstrates the use of the one-step transition controllers presented in [33, Sec. III]. A transition controller designed according to [33, Thm. 1] is able to effect a transition from the region of attraction of one walking controller to another by steering the state of the system using the same technical machinery used to render the zero dynamics invariant. The application of the transition controller is synchronized with swing leg touchdown.

For the experiment, the controller applied to RABBIT was transitioned between controllers at $0.1 \mathrm{~m} / \mathrm{s}$ intervals from 0.5 $\mathrm{m} / \mathrm{s}$ to $0.8 \mathrm{~m} / \mathrm{s}$ and then back from $0.8 \mathrm{~m} / \mathrm{s}$ to $0.5 \mathrm{~m} / \mathrm{s}$ twice (see Figure 22). The transitioning controllers were designed according to [34, Eqns. (12) and (13)]. The experiment lasted approximately 86 seconds during which RABBIT took 139 steps.

Figures 20 and 21 are plots of the actual and desired joint trajectories and the commanded control signals over a representative time interval of approximately twenty-six steps where the control was transitioned from $0.6 \mathrm{~m} / \mathrm{s}$ to $0.8 \mathrm{~m} / \mathrm{s}$. Note the change in the reference motion, $h_{d}$, with no visible difference in error, or in commanded control signal.

\subsubsection{Using event-based integral control to modify the fixed point}

In this fourth experiment, the same feedback used in the first experiment to induce walking at $0.7 \mathrm{~m} / \mathrm{s}$ was applied with the addition of an event-based PI control, developed in Appendix 2, to modify the steady state average walking rate from $0.7 \mathrm{~m} / \mathrm{s}$ to $0.6 \mathrm{~m} / \mathrm{s}$. The event-based control acts through modifications of the Bézier polynomial coefficients at double support events.

The event-based control was performed on the stance and swing leg relative angles, $q_{1}$ and $q_{2}$, which results in a change of the torso angle; see Figure 4(a). This was accomplished by setting $\delta \alpha$ to zero except for

$$
\delta \alpha_{j}^{i}=1, \text { for } i=1,2 \text { and } j=2, \ldots, M \text {. }
$$

The controller (71) with set-point $\eta^{*}=0.6$ and control gains $K_{I}=0.06$ and $K_{P}=0$ was applied on the 15 th step (at approximately 11 seconds). The proportional gain, $K_{P}$, was set to zero because of the noise introduced by the variance in step rate. The experiment lasted approximately 110 seconds during which RABBIT took 181 steps. Figure 26 gives the value of $w$ given in (71) versus time. Note that the ringing in $w$ and, consequently, in average walking rate is likely due to the integral gain being set too large; see Figure 27. Yet, if that is indeed the case, it still took over 50 steps for the average walking rate to converge to $0.6 \mathrm{~m} / \mathrm{s}$.

Figures 23-25 are plots of various quantities of interest for the entire experiment. Note the change in the reference motion, $h_{d}$, for $q_{1}$ and $q_{2}$ with no visible difference (in particular, no spikes) in commanded control signal. Figure 24 gives the torso angle change resulting from the changing of $q_{1}$ and $q_{2}$ (see the measurement conventions given in Figure 4(a)).

\subsubsection{Using event-based integral control to reject a pertur- bation}

In this fifth experiment, the same feedback used in the first experiment to induce walking at $0.7 \mathrm{~m} / \mathrm{s}$ was applied but with a $10 \mathrm{~kg}$ mass attached to the torso. This perturbation resulted in a shift of the average walking rate from $0.7 \mathrm{~m} / \mathrm{s}$ to approximately $0.85 \mathrm{~m} / \mathrm{s}$ (the change in average walking rate was determined in a separate experiment not reported here). The average walking rate of $0.7 \mathrm{~m} / \mathrm{s}$ was recovered using the event-based integral control described in the previous experiment but with $K_{I}=0.04$ and $\eta^{*}=0.7$ applied on the 14th step (at approximately 11 seconds). The experiment lasted approximately 95 seconds during which RABBIT took 164 steps.

Figure 29 gives the value of $w$ given in (71) versus time. Again, note that the ringing in $w$ and, consequently, in average walking rate (see Figure 30) is likely due to the integral gain being too large. The torso angle trace reflecting the action of the event-based integral control is given in Figure 28. This regulation of the torso angle by integral control was able to recover the $0.7 \mathrm{~m} / \mathrm{s}$ average walking rate (see Figure 30 ).

\subsubsection{Using event-based integral control to stop the robot}

In this sixth and final experiment, event-based integral control was used to stop RABBIT from a steady state average walking rate of $0.5 \mathrm{~m} / \mathrm{s}$. This was achieved by slowing the average walking rate of RABBIT to where it did not have enough kinetic energy to successfully complete a step; see Figure 33. The integral control described in the fourth experiment (Section 4.2.2) with $K_{I}=0.04$ and $\eta^{*}=0$ was applied on the 34th step (at approximately 29 seconds) and RABBIT was stopped by the 39th step (at approximately 34 seconds). After stopping, RABBIT rocked back and forth until all kinetic energy from walking was dissipated.

Figure 31 is a plot of the torso angle for a time interval including a portion of the steady state walking cycle and the stopping of RABBIT. The increase in the torso angle reflects the action of the integral control; see Figure 32. 


\section{Conclusion}

This paper presents the experimental validation of a novel framework for the control of walking in a class of planar bipeds using a 5-link biped prototype, RABBIT. The framework builds on previous work by formalizing an approach common to most schemes for the control of biped walking. That approach is to structure the control in such a way as to simplify the controller design process. The approach can be found, for example, in the regulation of angular momentum by Sano and Furusho [28], of total energy by Goswami, Espiau, and Keramane [10], of the robot's center of mass trajectory by Kajita and Tani [18], via virtual model control by Pratt et al. [24], [25], and of the ZMP by many [13], [21], [30], [35]. This framework is an attempt to give a rigorous formulation of this common approach.

After incorporation of three implementation issues, the experimental performance of the closed-loop system was found to be robust to both a sizable torso mass perturbation and aperiodic, short duration force perturbations applied to the torso. Two additional features of the framework were also validated. The first is the ability to compose two fixedrate walking controllers to obtain walking at several discrete walking rates with guaranteed stability during the transitions using a one-step transition controller. The second is the ability to regulate average walking rate to a continuum of values by modification of stride characteristics using an improvement to a previously published technique. The improved technique is given in Appendix 2.

The paper also interprets the action of the fixed-rate walking controllers as imposing virtual constraints and discusses how virtual constraints differ from physical constraints by means of a simple example. It is further discussed that the seeming equivalence of the dynamics that result from imposing virtual constraints to induce walking - the (swing phase) zero dynamics - and the dynamics of a length and inertia varying inverted pendulum does not hold.

\section{Acknowledgments}

The work of J.W. Grizzle and E.R. Westervelt was supported in part by NSF grants INT-9980227 and IIS-9988695.

\section{References}

[1] C.E. Bauby and A.D. Kuo. Active control of lateral balance in human walking. Journal of Biomechanics, 33(11):1433-40, November 2000.

[2] K. Berns. The walking machine catalogue, 2003. http://www . clawar.com/.

[3] G. Buche. ROBEA Home Page. http://www-lag.ensieg. inpg.fr/PRC-Bipedes/English/index . php.

[4] C. Chevallereau, G. Abba, Y. Aoustin, F. Plestan, E.R. Westervelt, C. Canduas-de Wit, and J.W. Grizzle. RABBIT: a testbed for advanced control theory. IEEE Control Systems Magazine, (to appear), October 2003. See [11] for a preprint.

[5] S. Diop, J.W. Grizzle, P.E. Moraal, and A. Stefanopoulou. Interpolation and numerical differentiation for observer design. In Proc. of the American Control Conference, Baltimore, MD, pages 1329-33, June 1994.

[6] J. Furusho and M. Masubuchi. Control of a dynamical biped locomotion system for steady walking. Journal of Dynamic Systems, Measurement and Control, 108:111-8, 1986.

[7] J. Furusho and A. Sano. Sensor-based control of a nine-link biped. International Journal of Robotics Research, 9(2):83-98, 1990.
[8] W. Goldsmith. Impact: the theory and physical behaviour of colliding solids. Arnold, London, 1960.

[9] A. Goswami. Postural stability of biped robots and the foot-rotation indicator (FRI) point. International Journal of Robotics Research, 18(6):523-33, June 1999.

[10] A. Goswami, B. Espiau, and A. Keramane. Limit cycles and their stability in a passive bipedal gait. In Proc. of the IEEE International Conference on Robotics and Automation, Minneapolis, MN, pages 246$51,1996$.

[11] J.W. Grizzle. Jessy Grizzle's publications, 2003. http: / / www . eecs . umich.edu/ grizzle/papers/robotics.html.

[12] J.W. Grizzle, G. Abba, and F. Plestan. Asymptotically stable walking for biped robots: Analysis via systems with impulse effects. IEEE Transactions on Automatic Control, 46:51-64, January 2001.

[13] K. Hirai, M. Hirose, Y. Haikawa, and T. Takenake. The development of Honda humanoid robot. In Proc. of the IEEE International Conference on Robotics and Automation, Leuven, Belgium, pages 1321-26, 1998.

[14] Q. Huang, S. Kajita, N. Koyachi, K. Kaneko, K. Yokoi, H. Arai, Komoriya K., and K. Tanie. A high stability, smooth walking pattern for a biped robot. In Proc. of the IEEE International Conference on Robotics and Automation, Detroit, MI, pages 65-71, 1999.

[15] Y. Hürmüzlü and D.B. Marghitu. Rigid body collisions of planar kinematic chains with multiple contact points. International Journal of Robotics Research, 13(1):82-92, 1994.

[16] A. Isidori. Nonlinear Control Systems: An Introduction. Springer-Verlag, Berlin, third edition, 1995.

[17] S. Kajita, F. Kanehiro, K. Kaneko, K. Fujiwara, K. Yokoi, and H. Hirukawa. A realtime pattern generator for biped walking. In Proc. of the IEEE International Conference on Robotics and Automation, Washington, D.C., pages 31-7, 2002.

[18] S. Kajita and K. Tani. Experimental study of biped dynamic walking. IEEE Control Systems Magazine, 16(1):13-9, February 1996.

[19] S. Kajita, T. Yamaura, and A. Kobayashi. Dynamic walking control of biped robot along a potential energy conserving orbit. IEEE Transactions on Robotics and Automation, 8(4):431-37, August 1992.

[20] A.D. Kuo. Stabilization of lateral motion in passive dynamic walking. International Journal of Robotics Research, 18(9):917-30, 1999.

[21] H. Lim, Y. Yamamoto, and A. Takanishi. Control to realize human-like walking of a biped humanoid robot. In Proc. of the IEEE International Conference on Systems, Man and Cybernetics, Computational Cybernetics and Simulations, Nashville, TN, pages 3271-76, June 2000.

[22] J.H. Park and K.D. Kim. Biped robot walking using gravity-compensated inverted pendulum mode and computed torque control. In Proc. of the IEEE International Conference on Robotics and Automation, Leuven, Belgium, pages 3528-33, 1998.

[23] F. Plestan, J.W. Grizzle, E.R. Westervelt, and G. Abba. Stable walking of a 7-dof biped robot. IEEE Transactions on Robotics and Automation, 19(4):653-68, August 2003.

[24] J.E. Pratt, M.C. Chee, A. Torres, P. Dilworth, and G.A. Pratt. Virtual model control: an intuitive approach for bipedal locomotion. International Journal of Robotics Research, 20(2):129-43, February 2001.

[25] J.E. Pratt and G.A. Pratt. Intuitive control of a planar bipedal walking robot. In Proc. of the IEEE International Conference on Robotics and Automation, Leuven, Belgium, pages 2014-21, 1998.

[26] M.H. Raibert. Legged robots that balance. MIT Press, Mass., 1986.

[27] A. Ruina and R. Pratap. Introduction to Statics and Dynamics. Oxford University Press. to be published, available at http: //www.tam . cornell.edu/ ruina/Book/.

[28] A. Sano and J. Furusho. Realization of natural dynamic walking using the angular momentum information. In Proc. of the IEEE International Conference on Robotics and Automation, Cincinnati, OH., pages 147681, May 1990.

[29] W.J. Schwind. Spring Loaded Inverted Pendulum Running: A Plant Model. PhD thesis, University of Michigan, 1998.

[30] M. Vukobratović, B. Borovac, D. Surla, and D. Stokic. Biped Locomotion. Springer-Verlag, Berlin, 1990.

[31] E.R. Westervelt. Eric Westervelt's publications, 2003. http: / / www . mecheng.osu.edu/ westerve/publications/.

[32] E.R. Westervelt. Toward a Coherent Framework for the Control of Planar Biped Locomotion. PhD thesis, University of Michigan, June 2003. available at [31].

[33] E.R. Westervelt, J.W. Grizzle, and C. Canudas de Wit. Switching and PI control of walking motions of planar biped walkers. IEEE Transactions on Automatic Control, 48(2), February 2003.

[34] E.R. Westervelt, J.W. Grizzle, and D.E. Koditschek. Hybrid zero dynamics of planar biped walkers. IEEE Transactions on Automatic Control, 48(1):42-56, January 2003. 
[35] J. Yamaguchi, E. Soga, S. Inoue, and A. Takanishi. Development of a bipedial humanoid robot: control method of whole body cooperative dynamic biped walking. In Proc. of the IEEE International Conference on Robotics and Automation, Detroit, MI, pages 368-74, 1999.

[36] H. Ye, A.N. Michel, and L. Hou. Stability theory for hybrid dynamical systems. IEEE Transactions on Automatic Control, 43(4):461-74, April 1998.

\section{Appendix 1 Hypotheses}

A complete list of hypotheses assumed for the $N$-link robot model and the desired walking gaits taken from [34] is enumerated.

Robot hypotheses: The robot is assumed to be:

RH1) comprised of $N$ rigid links with mass, connected by revolute joints with no closed kinematic chains;

RH2) planar, with motion constrained to the sagittal plane;

RH3) bipedal, with symmetric legs connected at a common point called the hip;

RH4) actuated at each joint;

RH5) unactuated at the point of contact between the stance leg and ground; and

RH6) modeled with $N-1$ relative angular coordinates, $\left(q_{1}, \ldots, q_{N-1}\right)$, plus one absolute angular coordinate, $q_{N}$.

Gait hypotheses: Conditions on the controller will be imposed and shown to ensure that the robot's consequent motion satisfies the following properties consistent with the intuitive notion of a simple walking gait:

GH1) there are alternating phases of single support and double support;

GH2) during the single support phase, the stance leg acts as a pivot joint; that is, throughout the contact, it can be guaranteed that the vertical component of the ground reaction force is positive and that the ratio of the horizontal component to the vertical component does not exceed the coefficient of static friction;

GH3) the double support phase is instantaneous and can be modeled as a rigid contact [15];

GH4) at impact, the swing leg neither slips nor rebounds;

GH5) in steady state, successive phases of single support are symmetric with respect to the two legs;

GH6) walking is from left to right, so that the swing leg starts from behind the stance leg and is placed strictly in front of the stance leg at impact.

RH1), RH2), and GH2) imply that the robot has $N$-DOF during the swing phase. RH4), RH5) and GH2) imply that when walking the robot has one degree of underactuation, i.e., one less control than DOF.

Impact model hypotheses: The impact model of [15] is used under the following assumptions:

IH1) the contact of the swing leg with the ground results in no rebound and no slipping of the swing leg;

$\mathrm{IH} 2$ ) at the moment of impact, the stance leg lifts from the ground without interaction;

IH3) the impact is instantaneous;

IH4) the external forces during the impact can be represented by impulses;
IH5) the impulsive forces may result in an instantaneous change in the velocities, but there is no instantaneous change in the configuration; and

IH6) the actuators cannot generate impulses and hence can be ignored during impact.

\section{Appendix 2 Event-based PI control of average walking rate}

The goal of this appendix is to design an event-based controller $^{10}$ that adjusts the parameters in the output (14) to achieve walking at a continuum of rates. This result is an extension to the event-based PI-control result given in [33]. The extension is nontrivial because of the difficulty of ensuring invariance of the hybrid zero dynamics in the presence of stepto-step stride-boundary parameter adjustments. The control technique was developed in response to the within-stride scheme's inability to effectively regulate average walking rate during experimentation. The controller design and analysis are based on the hybrid zero dynamics. A one-parameter curve will be defined in the set of parameters appearing in (14). Conditions will be identified so that this one-parameter curve will yield an effective control for the associated Poincaré map. Updating this control at each impact event of the walking cycle will yield a means to control average walking rate.

For a given controller ${ }^{11} \Gamma_{\alpha}$ satisfying the hypotheses of $\mathrm{CH} 2)-\mathrm{CH} 5)$ [12, Sec. IV-B] so that $Z_{\alpha}$ is invariant under the swing phase zero dynamics in closed loop with $\Gamma_{\alpha}$, the average walking rate is computed from the model (5) as follows. Let $P_{\alpha}: S \rightarrow S$ be the Poincaré return map and let $T_{I, \alpha}: T Q \rightarrow$ $\mathbb{R}_{\geq 0} \cup\{\infty\}$ be the time to impact function [34, Eqn. (81)]. Formally, the average walking rate is the (partial) map $\bar{\nu}_{\alpha}$ : $S \rightarrow \mathbb{R}_{\geq 0}$,

$$
\bar{\nu}_{\alpha}:=\frac{p_{2}^{\mathrm{h}} \circ P_{\alpha}}{T_{I, \alpha} \circ \Delta},
$$

where, $p_{2}^{\mathrm{h}}$, when evaluated on $S$, computes step length; see Figure 1. On the open subset $\tilde{S} \subset S$ where $0<T_{I, \alpha} \circ \Delta<\infty$ and the associated impacts are transversal to $S$, both $P_{\alpha}$ and $T_{I, \alpha} \circ \Delta$ are well-defined and continuous (see [12, Sec. III.B]). It follows that $\bar{\nu}_{\alpha}$ restricted to $\tilde{S}$ is continuous. Since $\Gamma_{\alpha}$ is continuous but not Lipschitz continuous, $\bar{\nu}_{\alpha}$ is not smooth on any open subset of $S$. However, if ${ }^{12} \alpha$ is a regular parameter value $^{13}$ of output (14) with $h_{0}, h_{d}$, and $\theta$ as in Section 3.1, giving rise to a hybrid zero dynamics that evolve on the associated zero dynamics manifold $Z_{\alpha}$, then $\bar{\nu}_{\alpha}$ restricted to $\tilde{S} \cap Z_{\alpha}$ depends smoothly on the states and the parameter values $\alpha$ used to define the outputs (14).

Let $A=\mathbb{R}^{(N-1) \times(M+1)}$ be the set of all Bézier polynomial coefficients, $\alpha$, for the output (14) with $h_{0}, h_{d}$, and $\theta$ as in Section 3.1. For this appendix, it is important to note that the

\footnotetext{
${ }^{10}$ That is, one that acts step-to-step with updates occurring at impacts

${ }^{11}$ In this appendix, to emphasize the dependence of quantities upon their associated Bézier polynomial parameters, the associated Bézier polynomial labels will be used as subscripts.

${ }^{12}$ The Bézier polynomial parameters, $\alpha_{k}^{i}$, are grouped into an $(N-1) \times$ $(M+1)$ matrix, $\alpha$, and denote the columns of $\alpha$ by $\alpha_{k}:=\left(\alpha_{k}^{1}, \ldots, \alpha_{k}^{N-1}\right)^{\prime}$.

${ }^{13}$ That is, the output corresponding to $\bar{\alpha}$ satisfies $\left.\left.\mathrm{HH} 1\right)-\mathrm{HH} 5\right)$.
} 
degrees of the Bézier polynomials in $h_{d}$ are fixed. Partition $A$, and, consequently, each $\alpha=\left[\alpha_{0}, \ldots, \alpha_{M}\right] \in A$, into two sets:

$$
\alpha_{\text {invar }}:=\left[\alpha_{0}, \alpha_{1}\right] \in A_{\text {invar }}:=\mathbb{R}^{(N-1) \times 2},
$$

the parameters chosen to render the swing phase zero dynamics invariant under the impact mapping and

$$
\alpha_{\text {free }}:=\left[\alpha_{2}, \ldots, \alpha_{M}\right] \in A_{\text {free }}:=\mathbb{R}^{(N-1) \times(M-1)},
$$

those freely chosen to affect the shape of the walking motion. Note that $A=A_{\text {invar }} \times A_{\text {free }}$ and $\alpha=\left[\alpha_{\text {invar }}, \alpha_{\text {free }}\right]$.

The natural geometric object to use in the analysis of eventbased, step-to-step parameter modifications is a fiber bundle. The fiber bundle structure will elucidate the interaction between parameter modifications and the evolution of a Poincarélike mapping, the flow map. To that end, define the trivial fiber bundle

$$
\pi: A \times T \mathcal{Q} \rightarrow A
$$

by $\pi(\alpha, x) \rightarrow \alpha$ for $\alpha \in A$ and $x \in T \mathcal{Q}$. Consider $\mathcal{S} \subset$ $A \times T \mathcal{Q}$ defined by

$$
\mathcal{S}:=\left\{(\alpha, x) \in A \times T \mathcal{Q} \mid \alpha \in A, p_{2}^{\mathrm{v}}(x)=0, p_{2}^{\mathrm{h}}(x)>0\right\} .
$$

which is a fiber bundle with base $A$ and fiber $\pi_{\mathcal{S}}^{-1}(\alpha)=S$ for each $\alpha \in \tilde{A} \subset A$ where $\pi_{\mathcal{S}}=\left.\pi\right|_{\mathcal{S}}$ and $\tilde{A}=\pi(\mathcal{S})$ is the set of parameters such that there exists at least one point where $p_{2}^{\mathrm{v}}(x)=0$ and $p_{2}^{\mathrm{h}}(x)>0$. Define $\bar{A} \subset \tilde{A}$ to be the set of regular parameters, i.e., for each $\bar{\alpha} \in \bar{A}$ the corresponding output satisfies HH1)-HH5). The set $\bar{A}$ is open since HH2), HH3), and HH5) are rank conditions ${ }^{14}$ and since condition HH4) requires a zero of a function that depends continuously on $\bar{\alpha}$ to remain in an open set. With $\bar{A}, \mathcal{Z} \subset A \times T \mathcal{Q}$ may be defined as

$$
\mathcal{Z}:=\left\{(\alpha, x) \in A \times T \mathcal{Q} \mid \alpha \in \bar{A}, h_{\alpha}(x)=0, L_{f} h_{\alpha}(x)=0\right\}
$$

which is a fiber bundle with base $\bar{A}$ and fiber $\pi_{\mathcal{Z}}^{-1}(\bar{\alpha})=Z_{\bar{\alpha}}$, $\bar{\alpha} \in \bar{A}$ and $\pi_{\mathcal{Z}}=\left.\pi\right|_{\mathcal{Z}}$. The fiber bundle $\mathcal{Z}$ corresponds to the set of parameters giving rise to well-defined swing phase zero dynamics along with the associated zero dynamics manifolds. Since, by assumption, for each $\bar{\alpha} \in \bar{A}$, the output satisfies HH5), the intersection $\mathcal{S} \cap \mathcal{Z}$ is also a fiber bundle with base $\bar{A} \subset A$ and fiber $\pi_{\mathcal{S} \cap \mathcal{Z}}^{-1}(\bar{\alpha})=S \cap Z_{\bar{\alpha}}, \bar{\alpha} \in \bar{A}$ where $\pi_{\mathcal{S} \cap \mathcal{Z}}=$ $\left.\pi\right|_{\mathcal{S} \cap \mathcal{Z}}$.

In what follows, the fiber bundle structure of $\mathcal{S} \cap \mathcal{Z}$ will permit the creation of event-based PI controllers which modify parameters step-to-step-even those that change $S \cap Z$ stepto-step. Let $\bar{\alpha} \in \bar{A}$ and suppose that $\delta \alpha \in \mathbb{R}^{(N-1) \times(M+1)}$ is such that

$$
\delta \alpha \neq 0 \quad \text { and } \quad(\delta \alpha)_{0}=(\delta \alpha)_{1}=0
$$

and that the function

$$
\hat{\alpha}_{\text {invar }}(\bar{\alpha}, v, w):=\left[\hat{\alpha}_{\text {invar }, 0}(\bar{\alpha}, v), \hat{\alpha}_{\text {invar }, 1}(\bar{\alpha}, v, w)\right]
$$

\footnotetext{
${ }^{14}$ An equivalent condition for a square matrix to be full rank is for its determinant to be nonzero. The determinant is a continuous function of matrix entries which, in the case of HH2), HH3), and HH5), are functions of $\bar{\alpha}$.
}

is defined to ensure invariance of the zero dynamics step-tostep. Then, for $v, w \in \mathbb{R}$ sufficiently small in magnitude

$$
\hat{\alpha}(\bar{\alpha}, v, w)=\left[\hat{\alpha}_{\text {invar }}(\bar{\alpha}, v, w), \bar{\alpha}_{\text {free }}\right]+w \delta \alpha
$$

is also regular. The following lemma will be used to calculate $\hat{\alpha}_{\text {invar }, 0}(\bar{\alpha}, v)$ and $\hat{\alpha}_{\text {invar }, 1}(\bar{\alpha}, v)$.

Lemma 1 (Achieving $\Delta\left(S \cap Z_{\alpha}\right) \subset Z_{\beta}$ ): Assume the existence of two outputs $h_{\alpha}$ and $h_{\beta}$ as defined above. Then, $h_{\beta} \circ \Delta\left(S \cap Z_{\alpha}\right)=0$ if, and only if,

$$
\left[\begin{array}{c}
\beta_{0} \\
\theta_{\beta}^{+}
\end{array}\right]=H \Delta_{q} H^{-1}\left[\begin{array}{c}
\alpha_{M_{\alpha}} \\
\theta_{\alpha}^{-}
\end{array}\right] .
$$

Moreover, if $\delta_{\text {zero, } \alpha} \neq 0$, then $L_{f} h_{\beta} \circ \Delta\left(S \cap Z_{\alpha}\right)=0$ if, and only if,

$$
\begin{aligned}
\beta_{1}=H_{0} \Delta_{\dot{q}} H^{-1}\left[\begin{array}{c}
\frac{M_{\alpha}}{\theta_{\alpha}^{-}-\theta_{\alpha}^{+}}\left(\alpha_{M_{\alpha}}-\alpha_{M_{\alpha}-1}\right) \\
1
\end{array}\right] \\
\\
\cdot \frac{\theta_{\beta}^{-}-\theta_{\beta}^{+}}{M_{\beta}} \frac{\kappa_{1, \alpha}\left(\theta_{\alpha}^{-}\right)}{\kappa_{1, \beta}\left(\theta_{\beta}^{+}\right)} \frac{1}{\delta_{\text {zero }, \alpha}}+\beta_{0}
\end{aligned}
$$

That is, if (61) and (62) hold, then $\Delta\left(S \cap Z_{\alpha}\right) \subset Z_{\beta}$.

Proof: The proof follows from direct calculation. The complete details are given in [32].

To ease the cumbersome notation, for the remainder of this appendix define $\bar{\alpha}_{v}:=\bar{\alpha}+v \delta \alpha$ and, similarly, $\bar{\alpha}_{w}:=\bar{\alpha}+w \delta \alpha$. Using this notation and Lemma $1, \hat{\alpha}_{\text {invar }, 0}$ and $\theta_{\hat{\alpha}}^{+}$may be calculated as

$$
\left[\begin{array}{c}
\hat{\alpha}_{\text {invar }, 0}(\bar{\alpha}, v) \\
\theta_{\hat{\alpha}}^{+}
\end{array}\right]:=H \Delta_{q} H^{-1}\left[\begin{array}{c}
\left(\bar{\alpha}_{v}\right)_{M} \\
\theta_{\bar{\alpha}_{v}}^{-}
\end{array}\right]
$$

to ensure that for each $x \in S$ if $h_{\bar{\alpha}}(x)=0$, then $h_{\bar{\alpha}} \circ \Delta(x)=$ 0 . If $\delta_{\text {zero }, \bar{\alpha}} \neq 0$, then $\hat{\alpha}_{\text {invar }, 1}$ may be calculated as

$$
\begin{gathered}
\hat{\alpha}_{\text {invar }, 1}(\bar{\alpha}, v, w):=H_{0} \Delta_{\dot{q}} H^{-1} \\
\cdot\left[\frac{M}{\theta_{\bar{\alpha}_{v}}^{-}-\theta_{\bar{\alpha}_{v}}^{+}}\left(\left(\bar{\alpha}_{v}\right)_{M}-\left(\bar{\alpha}_{v}\right)_{M-1}\right)\right] \\
\cdot \frac{\theta_{\bar{\alpha}_{w}}^{-}-\theta_{\bar{\alpha}_{w}}^{+}}{M} \frac{\kappa_{1, \bar{\alpha}_{v}}\left(\theta_{\bar{\alpha}_{v}}^{-}\right)}{\kappa_{1, \bar{\alpha}_{w}}\left(\theta_{\bar{\alpha}_{w}}^{+}\right)} \frac{1}{\delta_{\text {zero }, \bar{\alpha}_{v}}}+\hat{\alpha}_{\text {invar }, 0}(\bar{\alpha}, v)
\end{gathered}
$$

which together with $\hat{\alpha}_{\text {invar, } 0}$ ensures that for each $x \in S$ if $L_{f} h_{\bar{\alpha}}(x)=0$, then $L_{f} h_{\bar{\alpha}} \circ \Delta(x)=0$.

Assume that there exists some $\bar{\alpha}^{*} \in \bar{A}$ such that there exists a corresponding exponentially stable periodic orbit of the restricted Poincaré map, defined in Section 3,

$$
\rho_{\bar{\alpha}^{*}}: S \cap Z_{\bar{\alpha}^{*}} \rightarrow S \cap Z_{\bar{\alpha}^{*}} .
$$

Let $z_{\bar{\alpha}^{*}}^{*} \in \pi_{\mathcal{S} \cap \mathcal{Z}}^{-1}\left(\bar{\alpha}^{*}\right)$ be the corresponding fixed point of $\rho_{\bar{\alpha}^{*}}$. For $\bar{\alpha}^{*}, v$, and $w$ fixed, define the induced, restricted flow map

$$
\bar{\rho}_{\hat{\alpha}\left(\bar{\alpha}^{*}, v, w\right)}: \pi_{\mathcal{S} \cap \mathcal{Z}}^{-1} \circ \hat{\alpha}\left(\bar{\alpha}^{*}, \cdot, v\right) \rightarrow \pi_{\mathcal{S} \cap \mathcal{Z}}^{-1} \circ \hat{\alpha}\left(\bar{\alpha}^{*}, v, w\right)
$$

by $\bar{\rho}\left(z, \hat{\alpha}\left(\bar{\alpha}^{*}, v, w\right)\right): \varphi_{\hat{\alpha}\left(\bar{\alpha}^{*}, v, w\right)}\left(T_{I, \hat{\alpha}\left(\bar{\alpha}^{*}, v, w\right)}(z), z\right)$ where $\varphi_{\hat{\alpha}\left(\bar{\alpha}^{*}, v, w\right)}(t, z)$ is the maximal solution of the hybrid zero dynamics (22) with initial condition $z \in \pi_{\mathcal{S} \cap \mathcal{Z}}^{-1} \circ \hat{\alpha}\left(\bar{\alpha}^{*}, \cdot, v\right)$ at time $t_{0}=0$ associated with parameter $\hat{\alpha}\left(\bar{\alpha}^{*}, v, w\right)$. Unlike the restricted Poincaré map, $\rho$, which maps from a single fiber to 
itself, the induced, restricted flow map, $\bar{\rho}$, maps from one fiber (parameterized by $v \delta \alpha$ ) to another (parameterized by $w \delta \alpha$ ). The parameters $\delta \alpha, v$, and $w$ will now be used to implement event-based PI control on the induced, restricted flow map (66).

Define the single-input, single-output dynamic system on $\mathcal{S} \cap \mathcal{Z} \times \mathbb{R}^{2}$

$$
\begin{aligned}
z(k+1) & =\bar{\rho}(z(k), \alpha(k)) \\
\alpha(k) & =\hat{\alpha}\left(\bar{\alpha}^{*}, v(k), w(k)\right) \\
v(k+1) & =w(k) \\
\eta(k+1) & =\bar{\nu}(z(k), \alpha(k)) \\
y(k) & =\eta(k)
\end{aligned}
$$

with input $w \in \mathbb{R}$ and output $y \in \mathbb{R}$ equal to the average walking rate. It's linearization is

$$
\begin{aligned}
\delta z(k+1) & =a_{11} \delta z(k)+a_{12} \delta v(k)+b_{1} \delta w(k) \\
\delta \alpha(k) & =\delta \hat{\alpha}\left(\bar{\alpha}^{*}, v(k), w(k)\right) \\
\delta v(k+1) & =\delta w(k) \\
\delta \eta(k+1) & =a_{31} \delta z(k)+a_{32} \delta v(k)+b_{3} \delta w(k) \\
\delta y(k) & =\delta \eta(k)
\end{aligned}
$$

where

$$
\begin{aligned}
a_{11} & :=\frac{\partial \bar{\rho}}{\partial z}(z(k), \hat{\alpha}) & a_{31} & :=\frac{\partial \bar{\nu}}{\partial z}(z(k), \hat{\alpha}) \\
a_{12} & :=\frac{\partial \bar{\rho}}{\partial v}(z(k), \hat{\alpha}) & a_{32} & :=\frac{\partial \bar{\nu}}{\partial v}(z(k), \hat{\alpha}) \\
b_{1} & :=\frac{\partial \bar{\rho}}{\partial w}(z(k), \hat{\alpha}) & b_{3} & :=\frac{\partial \bar{\nu}}{\partial w}(z(k), \hat{\alpha}),
\end{aligned}
$$

$\hat{\alpha}=\hat{\alpha}\left(\bar{\alpha}^{*}, v(k), w(k)\right)$, and the right hand sides of (69) is evaluated at $z=z_{\bar{\alpha}^{*}}^{*}, v=0$, and $w=0$. The linearized system (68) is exponentially stable if, and only if, $\left|a_{11}\right|<1$. The DC-gain is non-zero if, and only if,

$$
a_{31}\left(b_{1}+a_{12}\right)+\left(a_{32}+b_{3}\right)\left(1-a_{11}\right) \neq 0 .
$$

Theorem 1: Let $\bar{\alpha}^{*} \in \bar{A}$ be a regular parameter value for which there exists an exponentially stable periodic orbit in $Z_{\bar{\alpha}^{*}}$. Denote the corresponding fixed point of the Poincare return map by $z_{\bar{\alpha}^{*}}^{*}$. Assume there exists $\delta \alpha$ satisfying (58) and such that the non-zero DC-gain condition (70) holds. Then, average walking rate can be regulated via PI control. In particular, there exist $\epsilon>0$, and scalars $K_{P}$ and $K_{I}$ such that for all $\eta^{*}$ such that $\left|\eta^{*}-\bar{\nu}\left(z_{\bar{\alpha}^{*}}^{*}, \bar{\alpha}^{*}\right)\right|<\epsilon$, the system consisting of (67) in closed-loop with the proportional plus integral controller

$$
\begin{aligned}
e(k+1) & =e(k)+\left(\eta^{*}-\eta(k)\right) \\
w(k) & =K_{P}\left(\eta^{*}-\eta(k)\right)+K_{I} e(k)
\end{aligned}
$$

has an exponentially stable equilibrium, and thus, when initialized sufficiently near the equilibrium, $\lim _{k \rightarrow \infty}\left(\eta^{*}-\eta(k)\right)=0$. Proof: The linear system (68) is exponentially stable because the exponential stability of the fixed-point $z_{\bar{\alpha}^{*}}^{*}$ implies that $\left|a_{11}\right|<1$. This, combined with the DC-gain being non-zero, implies the existence of a PI controller of the form

$$
\begin{aligned}
\delta e(k+1) & =\delta e(k)+\left(\delta \eta^{*}-\delta \eta(k)\right) \\
\delta w(k) & =K_{p}\left(\delta \eta^{*}-\delta \eta(k)\right)+K_{I} \delta e(k)
\end{aligned}
$$

such that the closed-loop system (68) with (72) is exponentially stable and satisfies $\lim _{k \rightarrow \infty}\left(\delta \eta^{*}-\delta \eta(k)\right)=0$, where $\delta \eta^{*}:=\left(\eta^{*}-\bar{\nu}\left(z_{\bar{\alpha}^{*}}^{*}, \bar{\alpha}^{*}\right)\right)$. Since the closed-loop of (68) with (72) is the linearization of (67) in closed-loop with (71), the result follows. 

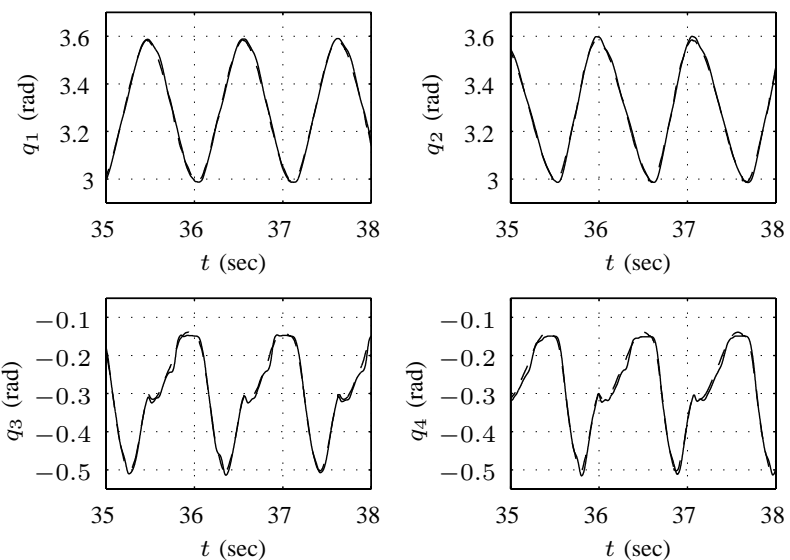

Fig. 12. Walking at $0.7 \mathrm{~m} / \mathrm{s}$ : joint angles versus time. Actual joint trajectories are solid and $h_{d, \text { is dashed. }}$
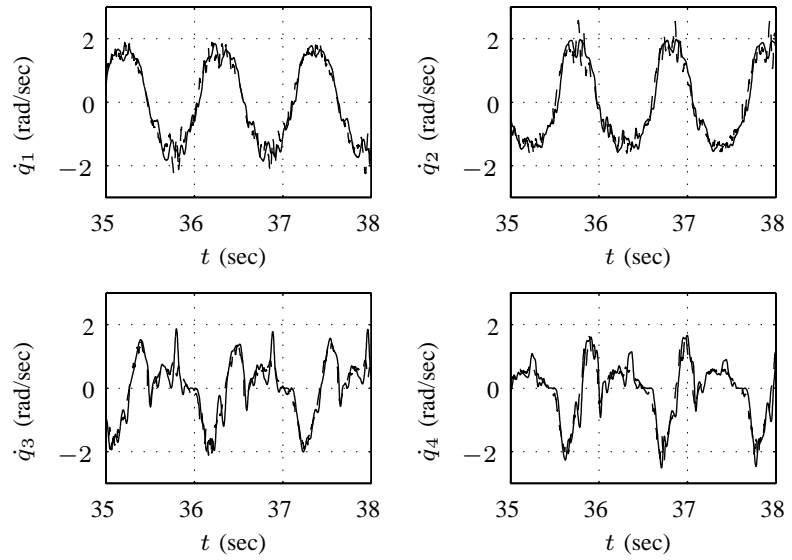

Fig. 13. Walking at $0.7 \mathrm{~m} / \mathrm{s}$ : joint velocities versus time. Actual joint trajectories are solid and $\left(\partial h_{d, \cdot} / \partial \hat{\theta}\right) \hat{\theta}$ is dashed.

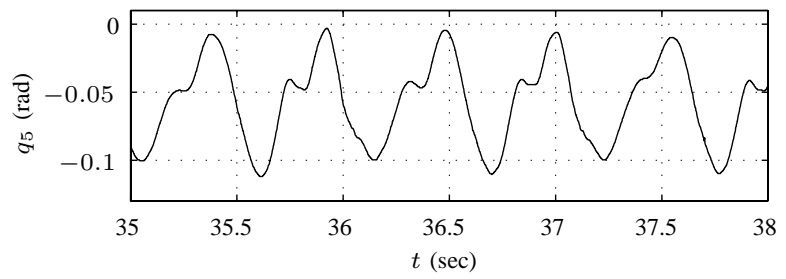

Fig. 14. Walking at $0.7 \mathrm{~m} / \mathrm{s}: q_{5}$ versus time.
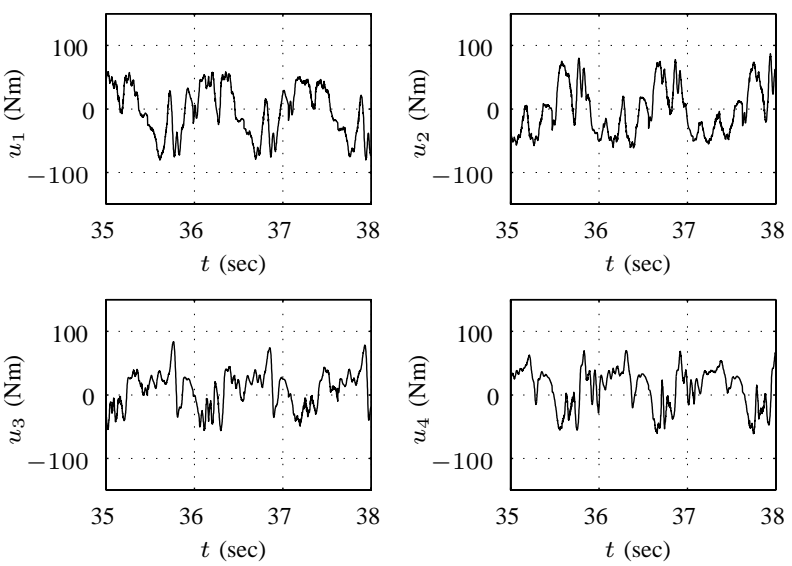

Fig. 15. Walking at $0.7 \mathrm{~m} / \mathrm{s}$ : control signals versus time.

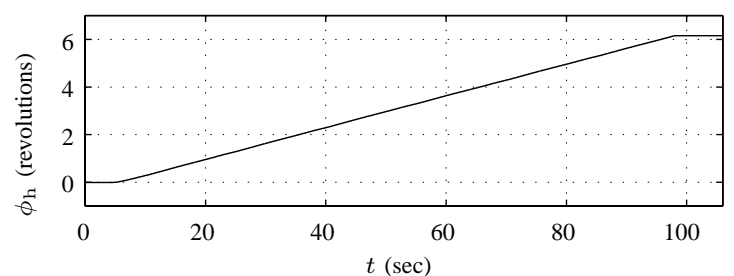

Fig. 16. Walking at $0.7 \mathrm{~m} / \mathrm{s}: \phi_{\mathrm{h}}$ versus time.
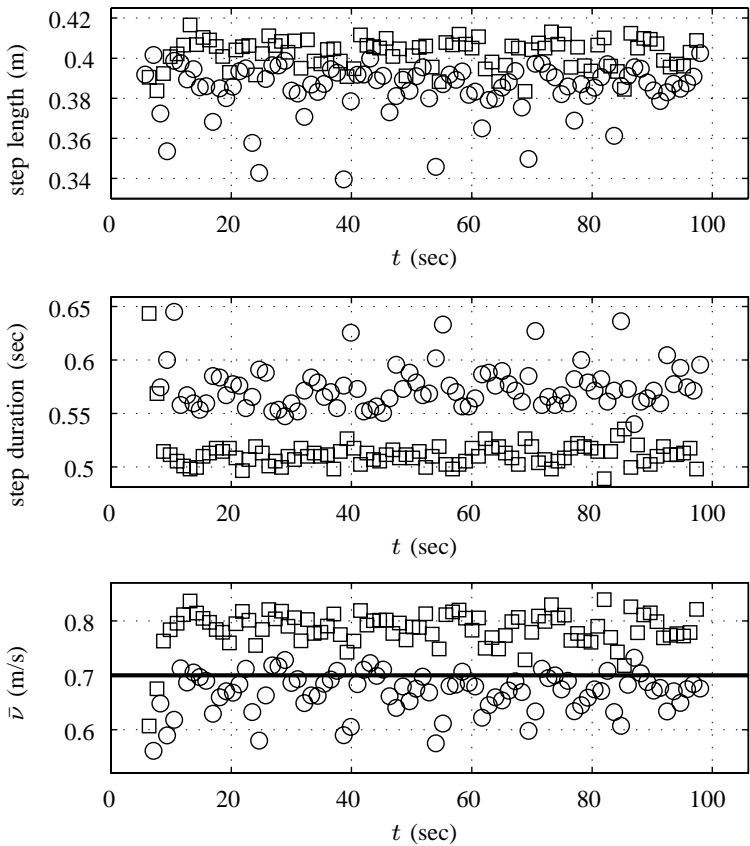

Fig. 17. Walking at $0.7 \mathrm{~m} / \mathrm{s}$ : step length, step duration, and average walking rate versus time. Circles represent steps taken by the outer leg, squares represent steps taken by the inner leg.
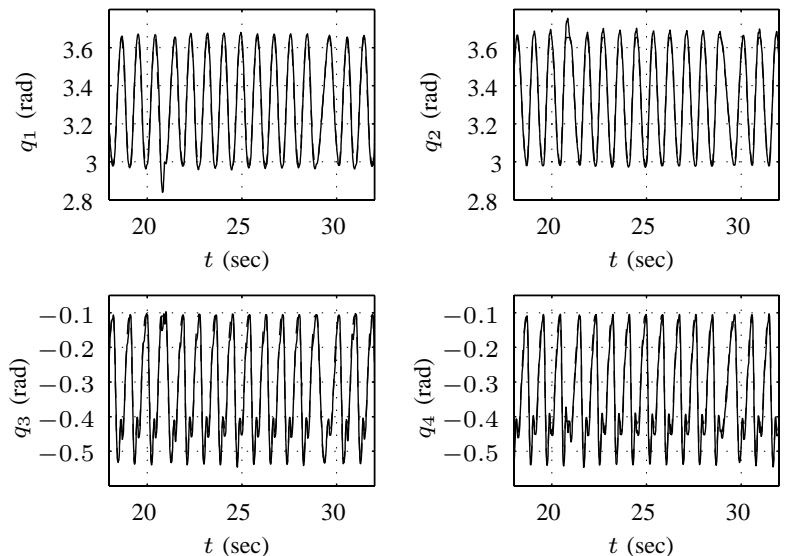

Fig. 18. Robustness demonstration: joint angles versus time. Actual joint trajectories are solid and $h_{d,}$, is dashed. 

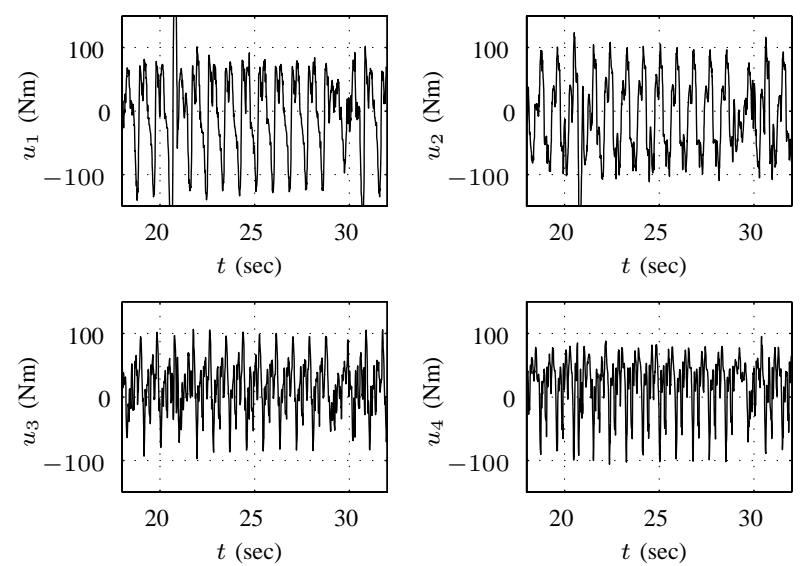

Fig. 19. Robustness demonstration: control signals versus time.
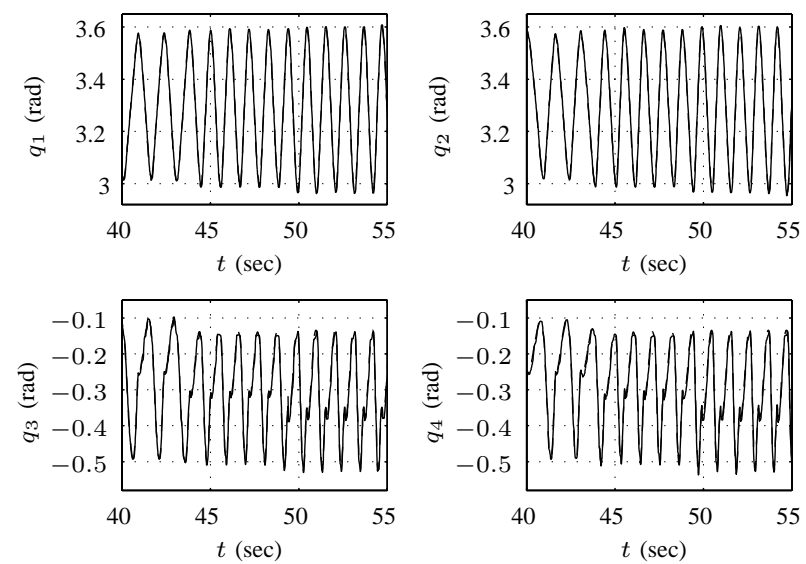

Fig. 20. Transitioning: joint angles versus time. Actual joint trajectories are

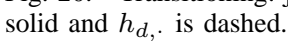
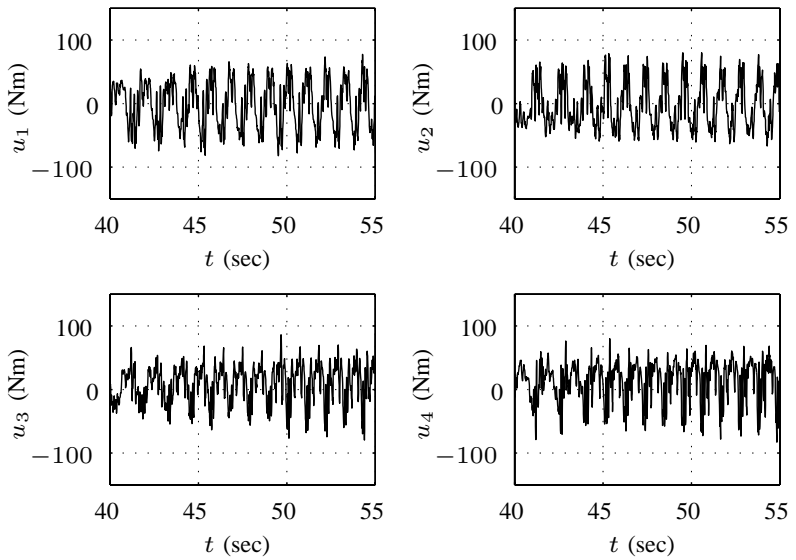

Fig. 21. Transitioning: control signals versus time.

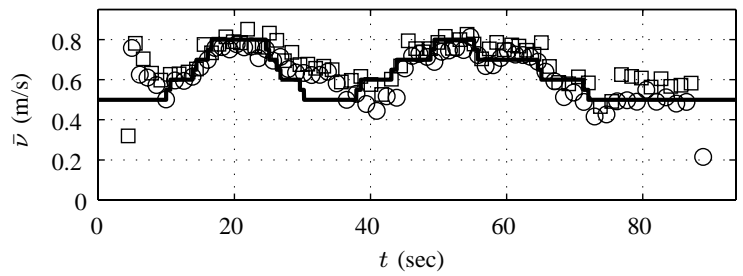

Fig. 22. Transitioning: average walking rate versus time. Circles represent steps taken by the outer leg, squares represent steps taken by the inner leg.
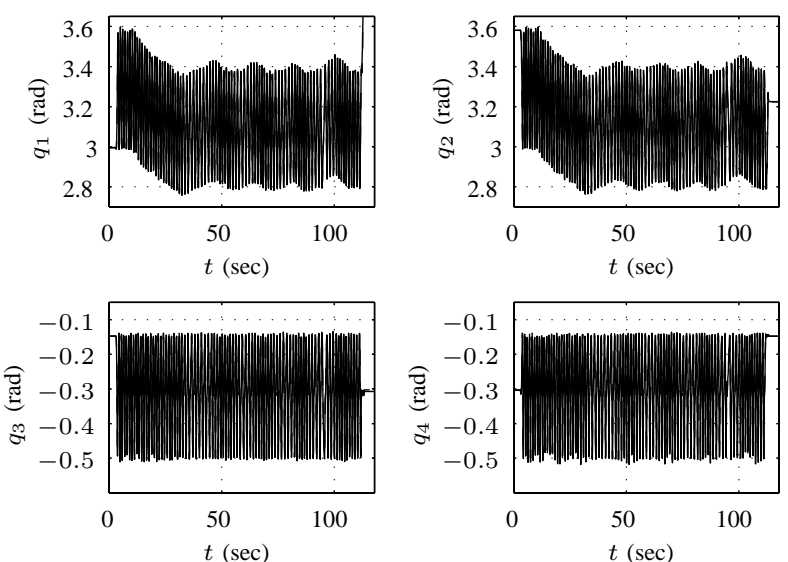

Fig. 23. I-control to change fixed point: joint angles versus time. Actual joint trajectories are solid and $h_{d,}$ is dashed.

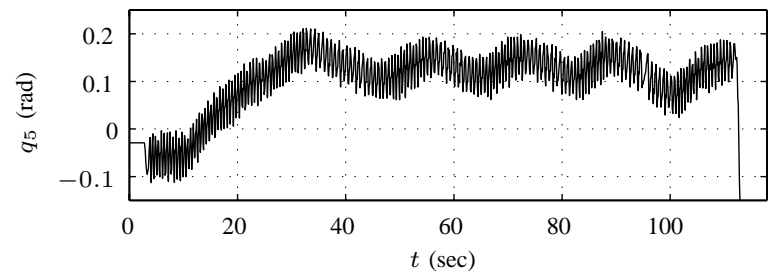

Fig. 24. I-control to change fixed point: $q_{5}$ versus time.
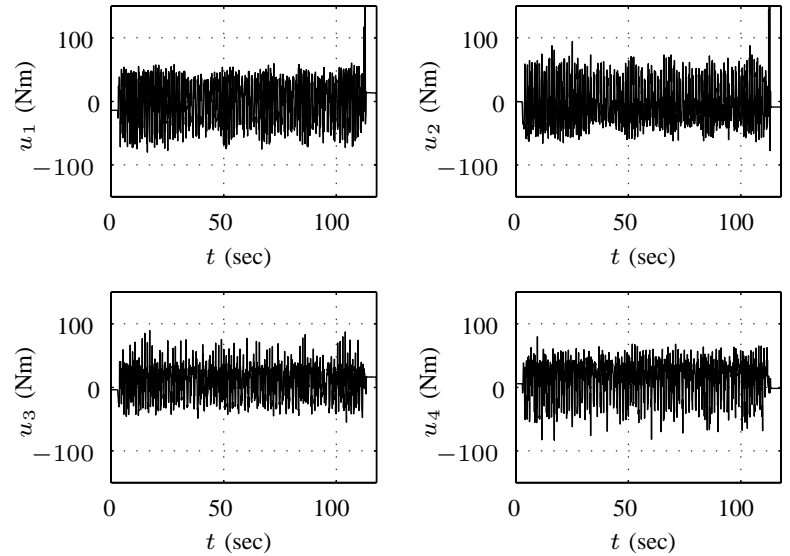

Fig. 25. I-control to change fixed point: control signals versus time.

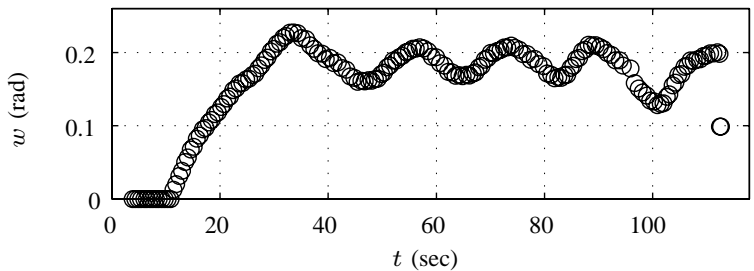

Fig. 26. I-control to change fixed point: $w$ versus time.

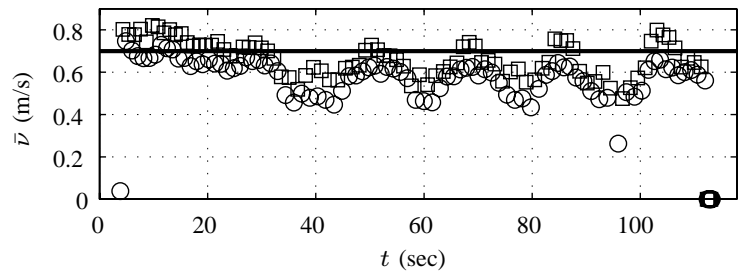

Fig. 27. I-control to change fixed point: average walking rate versus time. Circles represent steps taken by the outer leg, squares represent steps taken by the inner leg. 


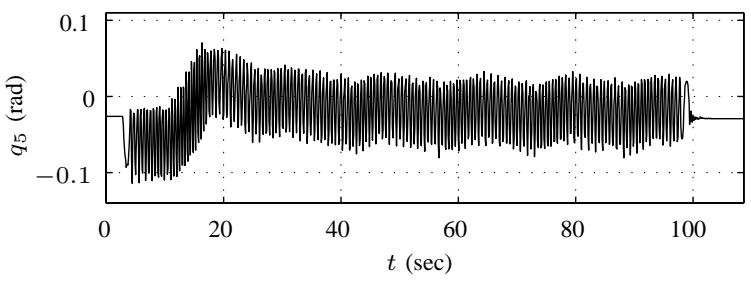

Fig. 28. I-control to reject a perturbation: $q_{5}$ versus time.

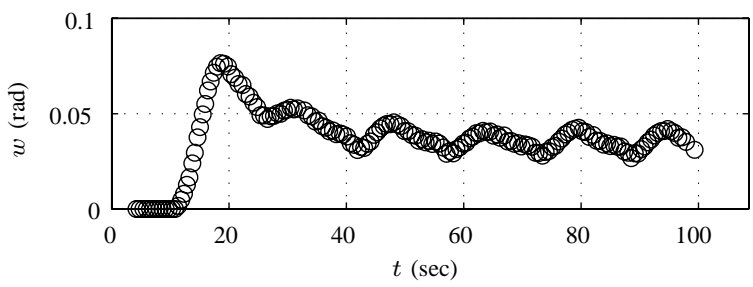

Fig. 29. I-control to reject a perturbation: $w$ versus time.

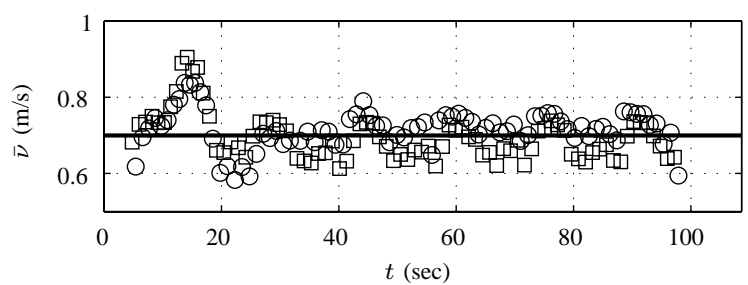

Fig. 30. I-control to reject a perturbation: average walking rate versus time. Circles represent steps taken by the outer leg, squares represent steps taken by the inner leg.

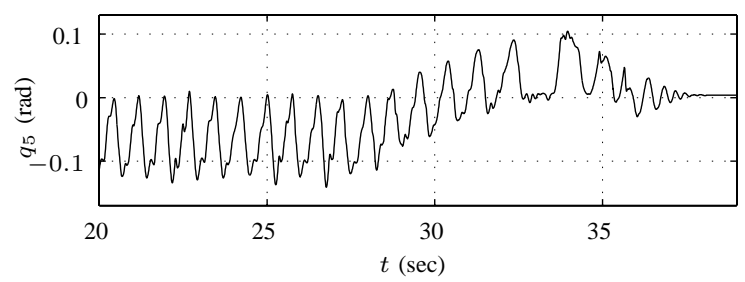

Fig. 31. I-control to stop the robot: $q_{5}$ versus time.

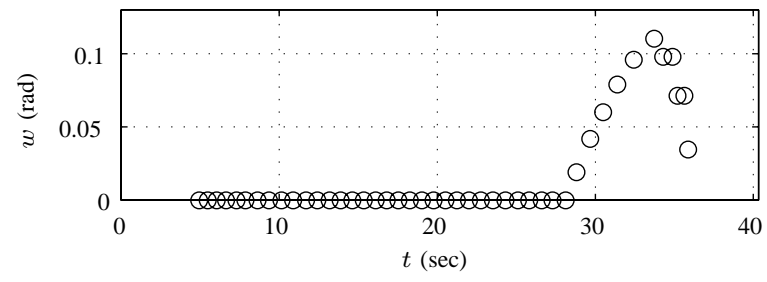

Fig. 32. I-control to stop the robot: $w$ versus time.

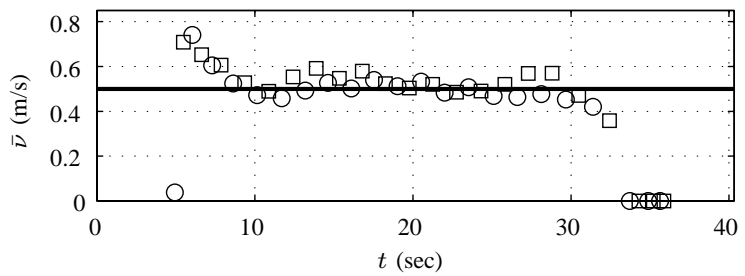

Fig. 33. I-control to stop the robot: average walking rate versus time. Circles represent steps taken by the outer leg, squares represent steps taken by the inner leg. 